\title{
FOURIER INTEGRALS AND SOBOLEV IMBEDDING ON REARRANGEMENT INVARIANT QUASI-BANACH FUNCTION SPACES
}

\begin{abstract}
Kwok-Pun Ho
The Hong Kong Institute of Education, Department of Mathematics and Information Technology 10 Lo Ping Road, Tai Po, Hong Kong, P. R. China; vkpho@ied.edu.hk

Abstract. We extend the mapping properties for the fractional integral operators, the convolution operators, the Fourier integral operators and the oscillatory integral operators to rearrangementinvariant quasi-Banach function spaces. We also generalize the Fourier restriction theorem and the Sobolev embedding theorem to rearrangement-invariant quasi-Banach function spaces. We obtain the above results by introducing two families of rearrangement-invariant quasi-Banach function spaces. Furthermore, these two families of rearrangement-invariant quasi-Banach function spaces also give us some embedding and interpolation results of Triebel-Lizorkin type spaces and Hardy type spaces built on rearrangement-invariant quasi-Banach function spaces.
\end{abstract}

\section{Introduction}

The main result of this paper aims to extend the studies of the Fourier integrals and Sobolev embedding to rearrangement-invariant quasi-Banach function spaces (r.i.q.B.f.s.). We achieve our result by introducing two families of rearrangementinvariant quasi-Banach function spaces.

The studies of Fourier integrals and Sobolev embeddings are two important topics of Fourier analysis and theory of function spaces. The development of these two topics is so deep and vast, therefore, it is impossible to give a detail review in this paper. For the study of the Fourier integrals on Lebesgue spaces, the reader is referred to the standard references $[30,31]$ and the references therein.

The action of operators on rearrangement-invariant quasi-Banach function spaces was first treated in [9]. For the Sobolev embedding of Sobolev spaces and TriebelLizorkin spaces, the reader is referred to [2, 32, 33].

The classical results for the Fourier integrals and Sobolev embedding are developed for Lebesgue spaces. The Lebesgue space has several generalizations such as the Lorentz spaces, the Lorentz-Karamata spaces and the Orlicz spaces and these generalizations are all members of rearrangement-invariant Banach function spaces. Notice that the Lorentz-Karamata spaces were first introduced in [9]. For a detailed reference on the rearrangement-invariant Banach function spaces, the reader is referred to [3].

Recently, by further developing of the extrapolation theory initiated by Rubio de Francia, Curbera, García-Cuerva, Martell and Pérez, [6, 7] extend some important results in Fourier analysis, such as the study of singular integral operators, to rearrangement-invariant Banach function spaces.

doi:10.5186/aasfm.2016.4157

2010 Mathematics Subject Classification: Primary 42B20, 42B35, 46B70, 46E30, 46E35, $42 \mathrm{~A} 96$.

Key words: Fourier integral operator, Sobolev embedding, oscillatory integrals, HausdorffYoung inequalities, restriction theorem, rearrangement-invariant, quasi-Banach function spaces, interpolation of operators, Triebel-Lizorkin spaces, Hardy spaces. 
The main theme of this paper is to extend the study of the Fourier integrals and Sobolev embedding to rearrangement-invariant quasi-Banach function spaces. Roughly speaking, we obtain these extensions by introducing two families of rearrangement-invariant quasi-Banach function spaces. We find that they are used to give the mapping properties for the fractional integral operators, the convolution operators, the Fourier integral operators and the oscillatory integral operators on r.i.q.B.f.s. These two families of function spaces are introduced by using an interpolation functor which is tailor-made for the rearrangement-invariant quasi-Banach function spaces.

It is well known that the Marcinkiewicz real interpolation functor $[3,4,5]$ is a powerful tool to extend the boundedness results of sublinear operators to Lebesgue spaces and Lorentz spaces. Our interpolation functor plays the same role as the Marcinkiewicz real interpolation with the Lorentz spaces replaced by the rearrangement-invariant quasi-Banach function spaces.

There are several celebrated theorems of using interpolation to extend results from the Lebesgue spaces to rearrangement-invariant Banach function spaces such as the Calderón theorem [3, Chapter 3, Theorem 5.7] and [3, Chapter 5, Theorem 1.19]. On the other hand, these theorems only give an abstract result on the mapping properties of linear operators on rearrangement-invariant Banach function spaces. They do not provide a recipe to precisely construct the function spaces involved in the mapping properties of the linear operators.

The interpolation functor used in this paper is not abstractly used to study function spaces. Our interpolation functor is defined explicitly by using the $K$ functional. Most importantly, our interpolation method is computable and generates rearrangement-invariant quasi-Banach function spaces in an explicit form in term of the non-decreasing rearrangement of Lebesgue measurable function. This is revealed by its applications on the convolution operators, the Fourier integral operators, the oscillatory integral operators and so on.

This paper is organized as follows. Section 2 presents those notions and notations used in this paper. We introduce the two families of r.i.q.B.f.s. in Section 3. Our interpolation functor is introduced in Section 4. It also establishes the action of this interpolation functor on Lebesgue spaces.

Sections 5, 6 and 7 present the main results of this paper. We establish the mapping properties of the convolution operators, the Fourier integral operators, the Fourier transform and the oscillatory integral operators on r.i.q.B.f.s. Section 6 also contains the Fourier restriction theorem on r.i.q.B.f.s. We study the Sobolev spaces associated with r.i.q.B.f.s. and present the corresponding Sobolev embedding in Section 7 .

The Sobolev embedding theorem is further extended to the Triebel-Lizorkin spaces associated with r.i.q.B.f.s. in Section 8. In this section, we also give the mapping properties of the fractional integral operator on Hardy spaces associated with r.i.q.B.f.s.

\section{Definitions and preliminaries}

For any Lebesgue measurable set $E \subseteq \mathbf{R}^{n}$, let $\mathcal{M}(E)$ be the set of Lebesgue measurable functions on $E$. Let $\mathcal{S}\left(\mathbf{R}^{n}\right)$ and $\mathcal{S}^{\prime}\left(\mathbf{R}^{n}\right)$ denote the class of Schwartz functions and tempered distributions, respectively.

For any and $f \in \mathcal{M}(E)$ and $s>0$, write

$$
d_{f}(s)=\left|\left\{x \in \mathbf{R}^{n}:|f(x)|>s\right\}\right|
$$


Fourier integrals and Sobolev embedding on rearrangement-invariant quasi-Banach function spaces 899

and

$$
f^{*}(t)=\inf \left\{s>0: d_{f}(s) \leq t\right\}, \quad t>0 .
$$

We call $f$ and $g$ are equimeasurable if $d_{f}(s)=d_{g}(s)$ for all $s>0$. We write $f \approx g$ if

$$
B f \leq g \leq C f
$$

for some constants $B, C>0$ independent of appropriate quantities involved in the expressions of $f$ and $g$.

We recall the definition of rearrangement-invariant quasi-Banach function space (r.i.q.B.f.s.) from [18, Definition 4.1].

Definition 2.1. A quasi-Banach space $X \subset \mathcal{M}\left(\mathbf{R}^{n}\right)$ is called a rearrangementinvariant quasi-Banach function space if there exists a quasi-norm $\rho_{X}: \mathcal{M}(0, \infty) \rightarrow$ $[0, \infty]$ satisfying

(1) $\rho_{X}(f)=0 \Leftrightarrow f=0$ a.e.,

(2) $|g| \leq|f|$ a.e. $\Rightarrow \rho_{X}(g) \leq \rho_{X}(f)$

(3) $0 \leq f_{n} \uparrow f$ a.e. $\Rightarrow \rho_{X}\left(f_{n}\right) \uparrow \rho_{X}(f)$,

(4) $\chi_{E} \in \mathcal{M}(0, \infty)$ and $|E|<\infty \Rightarrow \rho_{X}\left(\chi_{E}\right)<\infty$,

so that

$$
\|f\|_{X}=\rho_{X}\left(f^{*}\right), \quad \forall f \in X
$$

Write

$$
\bar{X}=\left\{g \in \mathcal{M}(0, \infty): \rho_{X}(g)<\infty\right\} .
$$

It is obvious that $\bar{X}$ is a r.i.q.B.f.s. on $(0, \infty)$. Recall that a Banach space $X \subset \mathcal{M}\left(\mathbf{R}^{n}\right)$ is a Banach function space if $\|\cdot\|_{X}$ is a norm and satisfies Items (1)-(3),

$$
\chi_{E} \in \mathcal{M}\left(\mathbf{R}^{n}\right) \text { and }|E|<\infty \Rightarrow \chi_{E} \in X
$$

and

$$
\chi_{E} \in \mathcal{M}\left(\mathbf{R}^{n}\right) \text { and } \quad|E|<\infty \Rightarrow \int_{E} f d x \leq C_{E} \rho_{X}(f),
$$

for some $C_{E}>0$. Moreover, $X$ is a rearrangement-invariant Banach function space (r.i.B.f.s) if $X$ is a Banach function space and for any equimeasurable functions $f$ and $g,\|f\|_{X}=\|g\|_{X}$.

Whenever $X$ is a r.i.B.f.s., the Luxemburg representation theorem [3, Chapter 2, Theorem 4.10] guarantees the existence of $\rho_{X}$ for $X$. Condition (2.3) assures that $\chi_{E}$ belongs to the dual space of $X$. On the other hand, a quasi-Banach space may not have a non-trivial dual spaces. For instance, $\left(L^{p}\right)^{*}=\{0\}, 0<p<1$. This is the reason why we do not impose (2.3) as a condition satisfied by r.i.q.B.f.s. When $X$ is a r.i.B.f.s., the associate space of $X$ is denoted by $X^{\prime}$. The reader is referred to [3, Chapter 1, Definitions 2.1 and 2.3] for the definition of associate space.

We give some classical examples of r.i.q.B.f.s., namely, the Lorentz spaces and the Orlicz spaces. For $0<p, q \leq \infty$, recall that the Lorentz space $L_{p, q}$ consists of all $f \in \mathcal{M}\left(\mathbf{R}^{n}\right)$ such that $\|f\|_{L_{p, q}}<\infty$ where

$$
\|f\|_{L_{p, q}}= \begin{cases}\left(\int_{0}^{\infty}\left(t^{\frac{1}{p}} f^{*}(t)\right)^{q} \frac{d t}{t}\right)^{\frac{1}{q}}, & q<\infty, \\ \sup _{t>0} t^{\frac{1}{p}} f^{*}(t), & q=\infty .\end{cases}
$$

The Lorentz spaces have been further generalized to the Lorentz-Karamata spaces, see [10, Section 3.4.1]. 
When $\Phi$ is a Young function, the Orlicz space $L^{\Phi}$ consists of those $f \in \mathcal{M}\left(\mathbf{R}^{n}\right)$ satisfying

$$
\|f\|_{L^{\Phi}}=\inf \left\{\lambda>0: \int_{\mathbf{R}^{n}} \Phi(|f(x)| / \lambda) d x \leq 1\right\}<\infty .
$$

For any $s \geq 0$ and $f \in \mathcal{M}(0, \infty)$, define $\left(D_{s} f\right)(t)=f(s t), t \in(0, \infty)$. Let $\left\|D_{s}\right\|_{\bar{X} \rightarrow \bar{X}}$ be the operator norm of $D_{s}$ on $\bar{X}$. We recall the definition of Boyd's indices for r.i.q.B.f.s. from [28].

Definition 2.2. Let $X$ be a r.i.q.B.f.s. on $\mathbf{R}^{n}$. Define the lower Boyd index of $X, p_{X}$, and the upper Boyd index of $X, q_{X}$, by

$$
\begin{aligned}
& p_{X}=\sup \left\{p>0: \exists C>0 \text { such that } \forall 0 \leq s<1,\left\|D_{s}\right\|_{\bar{X} \rightarrow \bar{X}} \leq C s^{-1 / p}\right\}, \\
& q_{X}=\inf \left\{q>0: \exists C>0 \text { such that } \forall 1 \leq s,\left\|D_{s}\right\|_{\bar{X} \rightarrow \bar{X}} \leq C s^{-1 / q}\right\},
\end{aligned}
$$

respectively.

The Boyd indices of the Lorentz space $L_{p, q}$ are $p_{L_{p, q}}=q_{L_{p, q}}=p$. For the Boyd indices of the Orlicz space $L^{\Phi}$, the reader is referred to [3].

Whenever $X$ is a r.i.B.f.s., the Boyd indices of $X$ given in the above definition coincide with the ones defined in [27, Volume II, Definition 2.b.1]. Moreover, when $X$ is a r.i.B.f.s., we have $1 \leq p_{X} \leq q_{X} \leq \infty$ and

$$
\frac{1}{p_{X}}+\frac{1}{q_{X^{\prime}}}=1 \text { and } \quad \frac{1}{q_{X}}+\frac{1}{p_{X^{\prime}}}=1
$$

For the proof of these identities, the reader is referred to [27, Volume II, Proposition 2.b.2]. Notice that the Boyd indices defined in [3, Chapter 3, Definition 5.12] are the reciprocals of the ones given in the above definition. For any r.i.q.B.f.s. $X$, according to Aoki-Rolewicz theorem [24, Theorem 1.3], there exists a $0<\kappa_{X} \leq 1$ such that $\rho_{X}^{\kappa_{X}}$ satisfies the triangle inequality.

We restate some supporting results from [3, Chapter 2]. Whenever $X$ is a r.i.B.f.s., from [3, Chapter 2, Proposition 4.2], we have

$$
\rho_{X}(f)=\sup \left\{\int_{0}^{\infty} f^{*}(s) g^{*}(s) d s: \rho_{X}^{\prime}(g) \leq 1\right\}
$$

where $\rho_{X}^{\prime}$ is the associate norm of $\rho_{X}$. The reader may consult [3, Chapter 1 , Definition 2.1] for the definition of associate norm. Moreover, [3, Chapter 2, Proposition 4.2] also assures that the norm $\rho_{X}^{\prime}$ is rearrangement-invariant on $\mathcal{M}(0, \infty)$. That is, $\rho_{X}^{\prime}\left(g^{*}\right)=\rho_{X}^{\prime}(g), \forall g \in \mathcal{M}(0, \infty)$.

We present the Hardy lemma in the following form.

Lemma 2.1. Let $\xi_{1}, \xi_{2} \in \mathcal{M}(0, \infty)$ with

$$
\int_{0}^{t} \xi_{1}(s) d s \leq \int_{0}^{t} \xi_{2}(s) d s, \quad \forall t>0 .
$$

If $\eta$ is a decreasing function on $(0, \infty)$, then

$$
\int_{0}^{\infty} \xi_{1}(t) \eta(t) d t \leq \int_{0}^{\infty} \xi_{2}(t) \eta(t) d t
$$

For the proof of the above lemma, the reader may consult [3, Chapter 2, Proposition 3.6]. 


\section{Two families of rearrangement-invariant quasi-Banach function spaces}

In this section, we introduce the two families of r.i.q.B.f.s. which are used to establish the mapping properties of the Fourier integrals and Sobolev embedding.

3.1. The family $\left\{\boldsymbol{X}_{\boldsymbol{\alpha}}\right\}$. We begin with the first family of r.i.q.B.f.s. which is related to the mapping properties of the fractional integral operators, the convolution operators and the Fourier integral operators. Moreover, it is also used in the Sobolev embedding of the Sobolev spaces built on r.i.B.f.s.

Definition 3.1. Let $\alpha \geq 0$. For any r.i.q.B.f.s. $X, X_{\alpha}$ consists of those $f \in$ $\mathcal{M}\left(\mathbf{R}^{n}\right)$ such that

$$
\|f\|_{X_{\alpha}}=\rho_{X}\left(t^{-\frac{\alpha}{n}} f^{*}(t)\right)<\infty
$$

Write $\rho_{X_{\alpha}}(g)=\rho_{X}\left(t^{-\frac{\alpha}{n}} g(t)\right), \forall g \in \mathcal{M}(0, \infty)$.

Obviously, from (2.1), we have $X_{0}=X$. For instance, when $X=L^{p}$, then $X_{\alpha}=$ $L_{\frac{n p}{n-p \alpha}, p}$. Thus, the set $X_{\alpha}$ can be considered as the Lorentz type space associated with $X$. We have the following fundamental properties of $X_{\alpha}$.

Proposition 3.1. Let $\alpha>0$ and $X$ be a r.i.q.B.f.s. If $0<p_{X} \leq q_{X}<\frac{n}{\alpha}$, then $X_{\alpha}$ is a r.i.q.B.f.s.

Proof. As $\rho_{X}$ fulfills (1)-(3) of Definition 2.1, $\rho_{X_{\alpha}}$ also satisfies (1)-(3) of Definition 2.1. Furthermore, for any Lebesgue measurable set $E=(0, b), b>1$,

$$
\rho_{X_{\alpha}}\left(\chi_{E}\right)=\rho_{X}\left(t^{-\frac{\alpha}{n}} \chi_{[0, b)}(t)\right) \leq \rho_{X}\left(t^{-\frac{\alpha}{n}} \chi_{[0,1)}(t)\right)+\rho_{X}\left(\chi_{[1, b)}(t)\right) .
$$

Obviously, $\rho_{X}\left(\chi_{[1, b)}(t)\right)<\infty$. It only needs to show that $\rho_{X}\left(t^{-\frac{\alpha}{n}} \chi_{[0,1)}(t)\right)<\infty$.

For any $k \in \mathbf{N} \cup\{0\}$, write $J_{k}=\left[2^{-k-1}, 2^{-k}\right)$. Since $[0,1)=\cup_{k=0}^{\infty} J_{k}$, we have

$$
\rho_{X}^{\kappa_{X}}\left(t^{-\frac{\alpha}{n}} \chi_{[0,1)}(t)\right) \leq \sum_{k=0}^{\infty} 2^{k \kappa_{X} \frac{\alpha}{n}} \rho_{X}^{\kappa_{X}}\left(\chi_{J_{k}}(t)\right)
$$

where $\kappa_{X}$ is the constant given by the Aoki-Rolewicz theorem [24, Theorem 1.3] for $\rho_{X}$. Moreover, $D_{2^{k}} \chi_{J_{0}}=\chi_{J_{k}}$. Thus, there exists a $q_{X}<q_{0}<\frac{n}{\alpha}$ such that

$$
\rho_{X}^{\kappa_{X}}\left(t^{-\frac{\alpha}{n}} \chi_{[0,1)}(t)\right) \leq \sum_{k=0}^{\infty} 2^{k \kappa_{X} \frac{\alpha}{n}} \rho_{X}^{\kappa_{X}}\left(D_{2^{k}} \chi_{J_{0}}(t)\right) \leq \sum_{k=0}^{\infty} 2^{k \kappa_{X}\left(\frac{\alpha}{n}-\frac{1}{q_{0}}\right)} \rho_{X}^{\kappa_{X}}\left(\chi_{J_{0}}\right)<\infty
$$

because $\rho_{X}^{\kappa_{X}}$ satisfies the triangle inequality.

We have $\rho_{X}\left(\chi_{J_{0}}\right)<\infty$ because $\rho_{X}$ fulfills Item (4) of Definition 2.1. Therefore, $\rho_{X}\left(t^{-\frac{\alpha}{n}} \chi_{[0,1)}(t)\right)<\infty$. That is, $\rho_{X_{\alpha}}$ fulfills $(4)$ of Definition 2.1. In view of $[3$, Chapter 2, (1.16)], we have

$$
(f+g)^{*}(t) \leq f^{*}(t / 2)+g^{*}(t / 2)
$$

Thus,

$$
\rho_{X}\left(t^{-\frac{\alpha}{n}}(f+g)^{*}(t)\right) \leq C\left(\rho_{X}\left(t^{-\frac{\alpha}{n}} f^{*}(t / 2)\right)+\rho_{X}\left(t^{-\frac{\alpha}{n}} g^{*}(t / 2)\right)\right) .
$$

Therefore, the condition $0<p_{X}$ assures that

$$
\|f+g\|_{X_{\alpha}} \leq C\left(\|f\|_{X_{\alpha}}+\|g\|_{X_{\alpha}}\right)
$$

for some $C>0$ independent of $f$ and $g$. Thus, $X_{\alpha}$ is a r.i.q.B.f.s.

Theorem 3.2. Let $\alpha>0$ and $X$ be a r.i.B.f.s. If $0<p_{X} \leq q_{X}<\frac{n}{\alpha}$, then $X_{\alpha}$ is a r.i.B.f.s. 
Proof. We first show that $\|\cdot\|_{X_{\alpha}}$ is a norm. Since $X$ is a r.i.B.f.s, by the definition of $X_{\alpha}$ and (2.4), for any $f, g \in \mathcal{M}\left(\mathbf{R}^{n}\right)$, we have

$$
\begin{aligned}
\|f+g\|_{X_{\alpha}} & =\rho_{X}\left(t^{-\frac{\alpha}{n}}(f+g)^{*}(t)\right) \\
& =\sup \left\{\int_{0}^{\infty} t^{-\frac{\alpha}{n}}(f+g)^{*}(t) h^{*}(t) d t: \rho_{X}^{\prime}(h) \leq 1\right\} .
\end{aligned}
$$

For any $h \in \mathcal{M}(0, \infty)$ with $\rho_{X}^{\prime}(h) \leq 1$, we find that $t^{-\frac{\alpha}{n}} h^{*}(t)$ is decreasing and $(f+g)^{* *} \leq f^{* *}+g^{* *}[3$, Chapter 2, Theorem 3.4]. According to the definition of $f^{* *}, \xi_{1}=(f+g)^{*}$ and $\xi_{2}=f^{*}+g^{*}$ satisfy (2.5). Thus, Lemma 2.1 and the Hölder inequality for $\rho_{X}[3$, Chapter 2, Corollary 4.3] assure that

$$
\begin{aligned}
\int_{0}^{\infty} t^{-\frac{\alpha}{n}}(f+g)^{*}(t) h^{*}(t) d t & \leq \int_{0}^{\infty} t^{-\frac{\alpha}{n}} f^{*}(t) h^{*}(t) d t+\int_{0}^{\infty} t^{-\frac{\alpha}{n}} g^{*}(t) h^{*}(t) d t \\
& \leq \rho_{X}\left(t^{-\frac{\alpha}{n}} f^{*}(t)\right) \rho_{X}^{\prime}\left(h^{*}(t)\right)+\rho_{X}\left(t^{-\frac{\alpha}{n}} g^{*}(t)\right) \rho_{X}^{\prime}\left(h^{*}(t)\right) \\
& \leq \rho_{X}\left(t^{-\frac{\alpha}{n}} f^{*}(t)\right)+\rho_{X}\left(t^{-\frac{\alpha}{n}} g^{*}(t)\right) .
\end{aligned}
$$

We obtain the last inequality because $\rho_{X}^{\prime}$ is a rearrangement-invariant function norm on $\mathcal{M}(0, \infty)$ and $h$ and $h^{*}$ are equimeasurable functions [3, Chapter 2, (1.19)]. Therefore, $\rho_{X}^{\prime}\left(h^{*}\right)=\rho_{X}^{\prime}(h) \leq 1$. Hence,

$$
\|f+g\|_{X_{\alpha}} \leq\|f\|_{X_{\alpha}}+\|g\|_{X_{\alpha}}
$$

Finally, for any Lebesgue measurable set $E$ with $|E|<\infty$, the Hölder inequality for $\rho_{X}[3$, Chapter 2, Corollary 4.3] guarantees that

$$
\int_{E} f(x) d x \leq \int_{0}^{|E|} f^{*}(t) d t \leq \rho_{X}\left(t^{-\frac{\alpha}{n}} f^{*}(t)\right) \rho_{X}^{\prime}\left(t^{\frac{\alpha}{n}} \chi_{[0,|E|]}\right) \leq|E|^{\frac{\alpha}{n}} \rho_{X}^{\prime}\left(\chi_{[0,|E|]}\right)\|f\|_{X_{\alpha}} .
$$

As $\rho_{X}^{\prime}\left(\chi_{[0,|E|]}\right)=\left\|\chi_{E}\right\|_{X^{\prime}}$, in view of [3, Chapter 1, Theorem 2.2], $\rho_{X}^{\prime}\left(\chi_{[0,|E|]}\right)<\infty$. Thus, $\|\cdot\|_{X_{\alpha}}$ fulfills (5) of Definition 2.1.

The subsequent result gives the relation of the Boyd indices of $X$ and $X_{\alpha}$.

Lemma 3.3. Let $\alpha>0$ and $X$ be a r.i.q.B.f.s. If $0<p_{X} \leq q_{X}<\frac{n}{\alpha}$, then

$$
\frac{1}{p_{X_{\alpha}}}=\frac{1}{p_{X}}-\frac{\alpha}{n} \text { and } \quad \frac{1}{q_{X_{\alpha}}}=\frac{1}{q_{X}}-\frac{\alpha}{n} .
$$

The proof of the above lemma follows from the definitions of the Boyd indices and $X_{\alpha}$. For brevity, we skip the proof. The main results for the family $\left\{X_{\alpha}\right\}$ are presented in Sections 5, 7 and 8 .

3.2. The family $\left\{\hat{\boldsymbol{X}}_{\beta}\right\}$. Next, we introduce the second family of r.i.q.B.f.s. It is associated with the mapping properties of the Fourier transform on r.i.q.B.f.s. For any $f \in \mathcal{S}^{\prime}\left(\mathbf{R}^{n}\right)$, we denote the Fourier transform of $f$ by $\hat{f}$.

Definition 3.2. Let $\beta>0$. For any r.i.q.B.f.s. $X$, the set $\hat{X}_{\beta}$ consists of all $f \in \mathcal{M}\left(\mathbf{R}^{n}\right)$ such that

$$
\|f\|_{\hat{X}_{\beta}}=\rho_{X}\left(t^{-1} f^{*}\left(t^{-\beta}\right)\right)<\infty .
$$

We write $\hat{X}_{1}=\hat{X}$.

Notice that $\hat{\hat{X}}=X$. This property for r.i.q.B.f.s. is consistent with the fact that $\hat{\hat{f}}(x)=f(-x), \forall f \in \mathcal{S}\left(\mathbf{R}^{n}\right)$. When $X=L^{p}, 1<p<\infty$, we have $\rho_{L^{p}}(g)=$ 
Fourier integrals and Sobolev embedding on rearrangement-invariant quasi-Banach function spaces 903 $\left(\int_{0}^{\infty} g(t)^{p} d t\right)^{\frac{1}{p}}$. Therefore,

$$
\|f\|_{\hat{X}}=\left(\int_{0}^{\infty}\left(t^{-1} f^{*}\left(t^{-1}\right)\right)^{p} d t\right)^{\frac{1}{p}}=\left(\int_{0}^{\infty} s^{p-1}\left(f^{*}(s)\right)^{p} \frac{d s}{s}\right)^{\frac{1}{p}}
$$

where we use the change of variable $s=t^{-1}$ in the last identity. Thus, by the definition of Lorentz spaces, we have

$$
\hat{L^{p}}=L_{p^{\prime}, p} .
$$

Thus, $\hat{X}$ can be considered as another extension of the notion of Lorentz spaces associated with r.i.q.B.f.s.

We now obtain a fundamental property for $\hat{X}_{\beta}$.

Theorem 3.4. Let $\beta>0$. If $X$ is a r.i.q.B.f.s. with $1<p_{X} \leq q_{X}<\infty$, then $\hat{X}_{\beta}$ is also a r.i.q.B.f.s.

Proof. Items (1)-(3) of Definition 2.1 are obviously fulfilled. It remains to show that $\|\cdot\|_{\hat{X}_{\beta}}$ is a quasi-norm and it satisfies Item (4) of Definition 2.1. As

$$
(f+g)^{*}(t) \leq f^{*}(t / 2)+g^{*}(t / 2), \quad \forall t>0,
$$

we obtain

$$
t^{-1}(f+g)^{*}\left(t^{-\beta}\right) \leq t^{-1} f^{*}\left(t^{-\beta} / 2\right)+t^{-1} g^{*}\left(t^{-\beta} / 2\right) .
$$

Thus, that $\|\cdot\|_{\hat{X}_{\beta}}$ is a quasi-norm follows from the assumption $q_{X}<\infty$.

To prove Item (4) of Definition 2.1, it suffices to consider $E=(0, b)$ for some $b>0$. Let $c=b^{-\beta}$. We find that

$$
\begin{aligned}
\rho_{X}^{\kappa_{X}}\left(t^{-1} \chi_{(0, b)}\left(t^{-\beta}\right)\right) & =\rho_{X}^{\kappa_{X}}\left(t^{-1} \chi_{(c, \infty)}(t)\right) \leq \sum_{k=0}^{\infty} \rho_{X}^{\kappa_{X}}\left(t^{-1} \chi_{\left(2^{k} c, 2^{k+1} c\right]}(t)\right) \\
& \leq C \sum_{k=0}^{\infty} 2^{-k \kappa_{X}} \rho_{X}^{\kappa_{X}}\left(\left(D_{2^{-k}} \chi_{(c, 2 c]}\right)(t)\right) \\
& \leq C \sum_{k=0}^{\infty} 2^{-k \kappa_{X}} 2^{\kappa_{X} k\left(\frac{1}{p_{X}}-\epsilon\right)} \rho_{X}^{\kappa_{X}}\left(\chi_{(c, 2 c]}(t)\right)
\end{aligned}
$$

Thus, Item (4) of Definition 2.1 follows from the assumption $1<p_{X}$.

The main results for $\left\{\hat{X}_{\beta}\right\}$ are established in Section 6 .

\section{Interpolation}

In this section, we present one of the tools used to obtain our main results in this paper. As the definition of interpolation functor involves the notion of category and compatible couples, for simplicity, we refer the reader to [32, Section 1.2] for details of category and compatible couples. We recall the definition of $K$-functional from [3, Section 3.1] and [32, Section 1.3.1].

Definition 4.1. Let $\left(X_{0}, X_{1}\right)$ be a compatible couple of quasi-normed spaces. For any $f \in X_{0}+X_{1}$, the $K$-functional is defined as

$$
K\left(f, t, X_{0}, X_{1}\right)=\inf \left\{\left\|f_{0}\right\|_{X_{0}}+t\left\|f_{1}\right\|_{X_{1}}: f=f_{0}+f_{1}\right\}
$$

where the infimum is taking over all $f=f_{0}+f_{1}$ for which $f_{i} \in X_{i}, i=0,1$.

We will write $K\left(f, t, X_{0}, X_{1}\right)$ as $K(f, t)$ if no confusion may occur. We introduce our interpolation functor in the following definition. 
Definition 4.2. Let $0<\theta, r<\infty$ and $X$ be a r.i.q.B.f.s. Let $\left(X_{0}, X_{1}\right)$ be a compatible couple of quasi-normed spaces. The space $\left(X_{0}, X_{1}\right)_{\theta, r, X}$ consists of all $f$ in $X_{0}+X_{1}$ such that

$$
\|f\|_{\left(X_{0}, X_{1}\right)_{\theta, r, X}}=\rho_{X}\left(t^{-\frac{1}{r}} K\left(f, t^{\frac{1}{\theta}}, X_{0}, X_{1}\right)\right)<\infty
$$

where $\rho_{X}$ is the quasi-norm given in (2.1).

According to [32, Section 1.2.2, Definition 1], $(\cdot, \cdot)_{\theta, r, X}$ is an interpolation functor if for any compatible couples $\left(X_{0}, X_{1}\right),(\cdot, \cdot)_{\theta, r, X}$ satisfies

(1) $X_{0} \cap X_{1} \hookrightarrow\left(X_{0}, X_{1}\right)_{\theta, r, X} \hookrightarrow X_{0}+X_{1}$.

(2) For any compatible couples $\left(X_{0}, X_{1}\right)$ and $\left(Y_{0}, Y_{1}\right)$, if the linear operator $L: X_{0}+$ $X_{1} \rightarrow Y_{0}+Y_{1}$ is bounded from $X_{i}$ to $Y_{i}, i=0,1$, then $L$ is also bounded from $\left(X_{0}, X_{1}\right)_{\theta, r, X}$ to $\left(Y_{0}, Y_{1}\right)_{\theta, r, X}$.

We now show that $(\cdot, \cdot)_{\theta, r, X}$ is indeed an interpolation functor.

Theorem 4.1. Let $0<\theta, r<\infty$ and $X$ be a r.i.q.B.f.s. with $0<p_{X} \leq q_{X}<\infty$. If $\frac{1}{q_{X}}+\frac{1}{\theta}>\frac{1}{r}$ and $r<p_{X}$, then $(\cdot, \cdot)_{\theta, r, X}$ is an interpolation functor. In addition, if $\left(X_{0}, X_{1}\right)$ and $\left(Y_{0}, Y_{1}\right)$ are compatible couples of quasi-normed spaces and $T$ is a linear operator such that

$$
\|T f\|_{Y_{i}} \leq M_{i}\|f\|_{X_{i}}, \quad i=0,1,
$$

then, for any $\epsilon$, there exists a constant $C_{\epsilon}>0$ independent of $M_{i}, i=0,1$ such that

$$
\|T f\|_{\left(Y_{0}, Y_{1}\right)_{\theta, r, X}} \leq C_{\epsilon} M\|f\|_{\left(X_{0}, X_{1}\right)_{\theta, r, X}}
$$

where

$$
M=\left(\frac{M_{1}}{M_{0}}\right)^{\frac{\theta}{r}} M_{0} \max \left(\left(\frac{M_{1}}{M_{0}}\right)^{-\frac{\theta}{p_{X}}+\epsilon},\left(\frac{M_{1}}{M_{0}}\right)^{-\frac{\theta}{q_{X}}-\epsilon}\right) .
$$

Proof. Let $\left(X_{0}, X_{1}\right)$ be a compatible couple of quasi-normed spaces. The embed$\operatorname{ding}\left(X_{0}, X_{1}\right)_{\theta, X} \hookrightarrow X_{0}+X_{1}$ is obvious because

$$
\min (1, t)\|f\|_{X_{0}+X_{1}} \leq K\left(f, t, X_{0}, X_{1}\right) .
$$

By the definition of $K$-functional, for any $f \in X_{0} \cap X_{1}$, we have

$$
K\left(f, t, X_{0}, X_{1}\right) \leq \min (1, t)\|f\|_{X_{0} \cap X_{1}} .
$$

Thus,

$$
\|f\|_{\left(X_{0}, X_{1}\right)_{\theta, r, X}}=\rho_{X}\left(t^{-\frac{1}{r}} K\left(f, t^{\frac{1}{\theta}}, X_{0}, X_{1}\right)\right) \leq\|f\|_{X_{0} \cap X_{1}} \rho_{X}\left(\min \left(t^{-\frac{1}{r}}, t^{\frac{1}{\theta}-\frac{1}{r}}\right)\right) .
$$

Since $\rho_{X}$ is a quasi-norm, Aoki-Rolewicz theorem [24, Theorem 1.3] assures that there exists a $\kappa_{X}$ such that $\rho_{X}^{\kappa_{X}}$ satisfies the triangle inequality. Thus,

$$
\begin{aligned}
& \rho_{X}^{\kappa_{X}}\left(\min \left(t^{-\frac{1}{r}}, t^{\frac{1}{\theta}-\frac{1}{r}}\right)\right) \leq \sum_{j=-\infty}^{0} 2^{j \kappa_{X}\left(\frac{1}{\theta}-\frac{1}{r}\right)} \rho_{X}^{\kappa_{X}}\left(\chi_{\left(2^{j-1}, 2^{j}\right]}\right)+\sum_{j=1}^{\infty} 2^{-\kappa_{X} \frac{j}{r}} \rho_{X}^{\kappa_{X}}\left(\chi_{\left(2^{j-1}, 2^{j}\right]}\right) \\
& =\sum_{j=-\infty}^{0} 2^{j \kappa_{X}\left(\frac{1}{\theta}-\frac{1}{r}\right)} \rho_{X}^{\kappa_{X}}\left(D_{2^{-(j-1)}} \chi_{(1,2]}\right)+\sum_{j=1}^{\infty} 2^{-\kappa_{X} \frac{j}{r}} \rho_{X}^{\kappa_{X}}\left(D_{2^{-(j-1)}} \chi_{(1,2]}\right) .
\end{aligned}
$$

Definition 2.2 assures that for any $\epsilon>0$, there exists a constant $C>0$ such that

$$
\begin{array}{ll}
\rho_{X}\left(D_{2^{-(j-1)}} \chi_{(1,2]}\right) \leq C 2^{j\left(\frac{1}{q_{X}}-\epsilon\right)} \rho_{X}\left(\chi_{(1,2]}\right), & j \in \mathbf{Z} \backslash \mathbf{N}, \\
\rho_{X}\left(D_{2^{-(j-1)}} \chi_{(1,2]}\right) \leq C 2^{j\left(\frac{1}{p_{X}}+\epsilon\right)} \rho_{X}\left(\chi_{(1,2]}\right), & j \in \mathbf{N} .
\end{array}
$$


Fourier integrals and Sobolev embedding on rearrangement-invariant quasi-Banach function spaces 905

As $\frac{1}{q_{X}}+\frac{1}{\theta}>\frac{1}{r}$ and $r<p_{X}$, when $\epsilon$ is sufficiently small, we have

$$
\begin{aligned}
\rho_{X}\left(\min \left(t^{-\frac{1}{r}}, t^{\frac{1}{\theta}-\frac{1}{r}}\right)\right) & =\sum_{j=-\infty}^{0} 2^{j \kappa_{X}\left(\frac{1}{\theta}-\frac{1}{r}+\frac{1}{q_{X}}-\epsilon\right)} \rho_{X}\left(\chi_{(1,2]}\right)+\sum_{j=1}^{\infty} 2^{j \kappa_{X}\left(-\frac{1}{r}+\frac{1}{p_{X}}+\epsilon\right)} \rho_{X}\left(\chi_{(1,2]}\right) \\
& <\infty .
\end{aligned}
$$

Hence, $X_{0} \cap X_{1} \hookrightarrow\left(X_{0}, X_{1}\right)_{\theta, r, X}$.

Let $\left(X_{0}, X_{1}\right)$ and $\left(Y_{0}, Y_{1}\right)$ be compatible couples. Suppose that the linear operator $T: X_{0}+X_{1} \rightarrow Y_{0}+Y_{1}$ satisfies (4.2). Write $K_{\theta, r}(t)=t^{-\frac{1}{r}} K\left(f, t^{\frac{1}{\theta}}, X_{0}, X_{1}\right)$. By the definition of $K$-functional, we find that

$$
\begin{aligned}
t^{-\frac{1}{r}} K\left(T(f), t^{\frac{1}{\theta}}, Y_{0}, Y_{1}\right) & \leq t^{-\frac{1}{r}} \inf _{\substack{f=f_{0}+f_{1} \\
f_{i} \in X_{i}, i=0,1}}\left(\left\|T\left(f_{0}\right)\right\|_{Y_{0}}+t^{\frac{1}{\theta}}\left\|T\left(f_{1}\right)\right\|_{Y_{1}}\right) \\
& \leq M_{0} t^{-\frac{1}{r}} K\left(f, \frac{M_{1}}{M_{0}} t^{\frac{1}{\theta}}, X_{0}, X_{1}\right) \\
& =\left(\frac{M_{1}}{M_{0}}\right)^{\frac{\theta}{r}} M_{0}\left(D_{\left(\frac{M_{1}}{M_{0}}\right)} K_{\theta, r}\right)(t) .
\end{aligned}
$$

We obtain

$$
\rho_{X}\left(t^{-\frac{1}{r}} K\left(T(f), t^{\frac{1}{\theta}}, Y_{0}, Y_{1}\right)\right) \leq\left(\frac{M_{1}}{M_{0}}\right)^{\frac{\theta}{r}} M_{0} \rho_{X}\left(\left(D_{\left(\frac{M_{1}}{M_{0}}\right)^{\theta}} K_{\theta, r}\right)(t)\right) .
$$

Hence, (4.3) and (4.4) follow from (4.1), (4.5), $0<p_{X} \leq q_{X}<\infty$ and Definition 2.2. Therefore, $(\cdot, \cdot)_{\theta, r, X}$ is an interpolation functor.

Let $X$ be a r.i.q.B.f.s. For any $0<p<\infty$, the $p$-convexification of $X, X^{p}$ is defined by $X^{p}=\left\{f:|f|^{p} \in X\right\}$. We equip $X^{p}$ with the quasi-norm $\|f\|_{X^{p}}=\left\||f|^{p}\right\|_{X}^{1 / p}$, see [27, Volume II, p. 53] and [29, Section 2.2].

Let $X$ be a r.i.q.B.f.s. The following theorem shows that $X_{\alpha}$ can be generated from the action of the functor $(\cdot, \cdot)_{\theta, r, X}$ on the Lebesgue spaces.

Theorem 4.2. Let $0 \leq \alpha<\infty, 0<p_{0}<p_{1}<\infty$ and $X$ be a r.i.q.B.f.s. with $0<p_{X} \leq q_{X}<\frac{n}{\alpha}$. Let $r, \theta$ satisfy

$$
\frac{1}{\theta}=\frac{1}{p_{0}}-\frac{1}{p_{1}} \text { and } \quad \frac{1}{r}=\frac{1}{p_{0}}+\frac{\alpha}{n} .
$$

Suppose that $p_{1}>q_{X}, p_{0}<p_{X}$ and

$$
\frac{1}{p_{1}}+\frac{\alpha}{n}<\frac{1}{q_{X}} \leq \frac{1}{p_{X}}<\frac{1}{p_{0}}+\frac{\alpha}{n}
$$

Then

$$
\left(L^{p_{0}}, L^{p_{1}}\right)_{\theta, r, X}=X_{\alpha}
$$

Proof. By (4.7), we have

$$
\begin{aligned}
\frac{1}{q_{X}}+\frac{1}{\theta} & =\frac{1}{q_{X}}+\frac{1}{p_{0}}-\frac{1}{p_{1}}=\frac{1}{q_{X}}+\frac{1}{r}-\frac{\alpha}{n}-\frac{1}{p_{1}}>\frac{1}{r}, \\
\frac{1}{p_{X}} & <\frac{1}{p_{0}}+\frac{\alpha}{n}=\frac{1}{r} .
\end{aligned}
$$

Theorem 4.1 assures that the functor $(\cdot, \cdot)_{\theta, p_{0}, X}$ is well defined. 
The Holmstedt formulas for the $K$-functionals of Lebesgue spaces [22] yield $t^{-\frac{1}{r}} K\left(f, t^{\frac{1}{\theta}}, L^{p_{0}}, L^{p_{1}}\right) \approx t^{-\frac{1}{p_{0}}-\frac{\alpha}{n}}\left(\int_{0}^{t}\left(f^{*}(s)\right)^{p_{0}} d s\right)^{\frac{1}{p_{0}}}+t^{-\frac{1}{p_{1}}-\frac{\alpha}{n}}\left(\int_{t}^{\infty}\left(f^{*}(s)\right)^{p_{1}} d s\right)^{\frac{1}{p_{1}}}$.

Thus,

$$
\begin{aligned}
& \rho_{X}\left(t^{-\frac{1}{r}} K\left(f, t^{\frac{1}{\theta}}, L^{p_{0}}, L^{p_{1}}\right)\right) \\
& \leq C \rho_{X}\left(t^{-\frac{1}{p_{0}}-\frac{\alpha}{n}}\left(\int_{0}^{t}\left(f^{*}(s)\right)^{p_{0}} d s\right)^{\frac{1}{p_{0}}}\right)+C \rho_{X}\left(t^{-\frac{1}{p_{1}}-\frac{\alpha}{n}}\left(\int_{t}^{\infty}\left(f^{*}(s)\right)^{p_{1}} d s\right)^{\frac{1}{p_{1}}}\right) \\
& =C \rho_{X}\left(t^{-\frac{\alpha}{n}}\left(\int_{0}^{1}\left(f^{*}(t s)\right)^{p_{0}} d s\right)^{\frac{1}{p_{0}}}\right)+C \rho_{X}\left(t^{-\frac{\alpha}{n}}\left(\int_{1}^{\infty}\left(f^{*}(t s)\right)^{p_{1}} d s\right)^{\frac{1}{p_{1}}}\right) \\
& =I+I I
\end{aligned}
$$

for some $C>0$.

We first consider $I$. As $f^{*}$ is non-increasing, we find that

$$
I \leq C \rho_{X^{\frac{1}{p_{0}}}}^{\frac{1}{p_{0}}}\left(t^{-\frac{p_{0} \alpha}{n}} \int_{0}^{1}\left(f^{*}(t s)\right)^{p_{0}} d s\right) \leq C \rho_{X^{\frac{1}{p_{0}}}}^{\frac{1}{p_{0}}}\left(t^{-\frac{p_{0} \alpha}{n}} \sum_{j=-\infty}^{0} 2^{j-1}\left(f^{*}\left(2^{j-1} t\right)\right)^{p_{0}}\right) .
$$

Write $f_{\alpha}^{*}(t)=t^{-\frac{\alpha}{n}} f^{*}(t)$. Then, $\rho_{X}\left(f_{\alpha}^{*}\right)=\rho_{X_{\alpha}}(f)$. The Aoki-Rolewicz theorem [24, Theorem 1.3] offers a $0<\kappa_{0} \leq 1$ such that $\rho_{X^{\frac{\kappa_{0}}{p_{0}}}}$ satisfies the triangle inequality. Thus,

$$
\begin{aligned}
I^{p_{0} \kappa_{0}} & \leq C \rho_{X^{\frac{1}{p_{0}}}}^{\kappa_{0}}\left(t^{-\frac{p_{0} \alpha}{n}} \sum_{j=-\infty}^{0} 2^{j-1}\left(f^{*}\left(2^{j-1} t\right)\right)^{p_{0}}\right) \\
& \leq C \sum_{j=-\infty}^{0} 2^{(j-1)\left(1+\frac{p_{0} \alpha}{n}\right) \kappa_{0}} \rho_{X^{\frac{1}{p_{0}}}}^{\kappa_{0}}\left(\left(D_{2^{j-1}} f_{\alpha}^{*}\right)^{p_{0}}\right) \\
& =C \sum_{j=-\infty}^{0} 2^{(j-1)\left(1+\frac{p_{0} \alpha}{n}\right) \kappa_{0}}\left(\rho_{X}\left(D_{2^{j-1}} f_{\alpha}^{*}\right)\right)^{p_{0} \kappa_{0}} .
\end{aligned}
$$

According to Definition 2.2, for any $\epsilon>0$, there exists a constant $C>0$ such that

$$
I^{p_{0} \kappa_{0}} \leq C \sum_{j=-\infty}^{0} 2^{(j-1)\left(1+\frac{p_{0} \alpha}{n}\right) \kappa_{0}} 2^{-\frac{(j-1) p_{0} \kappa_{0}}{p_{X}-\epsilon}}\left(\rho_{X}\left(f_{\alpha}^{*}\right)\right)^{p_{0} \kappa_{0}}
$$

In view of (4.7), when $\epsilon$ is sufficiently small, we find that $1+\frac{p_{0} \alpha}{n}-\frac{p_{0}}{p_{X}-\epsilon}>0$. Consequently,

$$
I \leq C \rho_{X_{\alpha}}\left(f^{*}\right)=C\|f\|_{X_{\alpha}} .
$$

Next, we deal with $I I$. Similar to the proof of $I$, the Aoki-Rolewicz theorem [24, Theorem 1.3] provides a $0<\kappa_{1} \leq 1$ such that $\rho_{X^{\frac{1}{p_{1}}}}^{\kappa_{1}}$ fulfills the triangle inequality. Consequently,

$$
I I^{p_{1} \kappa_{1}} \leq C \rho_{X^{\frac{1}{p_{1}}}}^{\kappa_{1}}\left(t^{-\frac{\alpha p_{1}}{n}}\left(\sum_{j=0}^{\infty}\left(f^{*}\left(2^{j} t\right)\right)^{p_{1}} 2^{j}\right)\right) \leq C \sum_{j=0}^{\infty} 2^{j p_{1} \kappa_{1}\left(\frac{1}{p_{1}}+\frac{\alpha}{n}\right)} \rho_{X}^{\kappa_{1} p_{1}}\left(D_{2^{j}} f_{\alpha}^{*}\right) .
$$


By the definition of $q_{X}$, we find that for any $\epsilon>0$, there exists a constant $C>0$ such that

$$
I I^{p_{1} \kappa_{1}} \leq C \sum_{j=0}^{\infty} 2^{j p_{1} \kappa_{1}\left(\frac{1}{p_{1}}+\frac{\alpha}{n}\right)} 2^{-\frac{j p_{1} \kappa_{1}}{q_{X}+\epsilon}} \rho_{X}^{\kappa_{X}}\left(f_{\alpha}^{*}\right) .
$$

Thus, (4.7) guarantees that for any sufficiently small $\epsilon>0$, there exists a constant $C>0$ such that

$$
I I \leq C \rho_{X_{\alpha}}\left(f^{*}\right)=C\|f\|_{X_{\alpha}} .
$$

Therefore, (4.8) and (4.9) conclude that $X_{\alpha} \hookrightarrow\left(L^{p_{0}}, L^{p_{1}}\right)_{\theta, r, X}$.

For the reverse embedding, we get

$$
\begin{aligned}
\rho_{X}\left(t^{-\frac{1}{r}} K\left(f, t^{\frac{1}{\theta}}, L^{p_{0}}, L^{p_{1}}\right)\right) & \geq C \rho_{X}\left(t^{-\frac{1}{p_{0}}-\frac{\alpha}{n}}\left(\int_{0}^{t}\left(f^{*}(s)\right)^{p_{0}} d s\right)^{\frac{1}{p_{0}}}\right) \\
& \geq C \rho_{X}\left(t^{-\frac{1}{p_{0}}-\frac{\alpha}{n}} t^{\frac{1}{p_{0}}} f^{*}(t)\right)=C \rho_{X_{\alpha}}\left(f^{*}\right)
\end{aligned}
$$

because $f^{*}$ is non-increasing. That is, the embedding $\left(L^{p_{0}}, L^{p_{1}}\right)_{\theta, r, X} \hookrightarrow X_{\alpha}$ is valid.

In particular, when $\alpha=0$, we have the following interpolation theorem for r.i.q.B.f.s.

Corollary 4.3. Let $0<p_{0}<p_{1}<\infty$ and $X$ be a r.i.q.B.f.s. with $0<p_{X} \leq$ $q_{X}<\infty$. Suppose that $p_{0}$ and $p_{1}$ satisfy $p_{1}>q_{X}, p_{0}<p_{X}$ and $\frac{1}{\theta}=\frac{1}{p_{0}}-\frac{1}{p_{1}}$. Then

$$
\left(L^{p_{0}}, L^{p_{1}}\right)_{\theta, p_{0}, X}=X .
$$

Some important examples of r.i.q.B.f.s. are Orlicz spaces and Lorentz-Karamata spaces. There are some interpolation functors generates these function spaces as the interpolation spaces of $L^{p}$. For the Orlicz spaces, the reader may consult [14]. For the Lorentz-Karamata spaces, the reader is referred to $[13,19]$. The above corollary shows that the interpolation functor introduced in this paper provides an unified method for generating Orlicz spaces and Lorentz-Karamata spaces from Lebesgue spaces.

\section{Convolutions and Fourier integral operators}

In this section, we present the first group of the main results of this paper on the fractional integral operators, the convolution operator and Fourier integral operators. We begin with the mapping property of the fractional integral operator

$$
\left(I_{\alpha} f\right)(x)=\int_{\mathbf{R}^{n}} \frac{f(y)}{|x-y|^{n-\alpha}} d y
$$

where $0<\alpha<n$.

Theorem 5.1. Let $0<\alpha<n$ and $X$ be a r.i.q.B.f.s. If $1<p_{X} \leq q_{X}<\frac{n}{\alpha}$, then

$$
\left\|I_{\alpha}(f)\right\|_{X_{\alpha}} \leq C\|f\|_{X}, \quad \forall f \in X
$$

for some $C>0$.

Proof. It is well-known that $I_{\alpha}: L^{s} \rightarrow L^{q}$ is bounded when

$$
1<s<\frac{n}{\alpha} \text { and } \quad \frac{1}{s}=\frac{1}{q}+\frac{\alpha}{n} .
$$


Since $1<p_{X} \leq q_{X}<\frac{n}{\alpha}$, there exist $s_{1}$, $s_{0}$ such that $q_{X}<s_{1}<\frac{n}{\alpha}$ and $1<s_{0}<p_{X}$. The mappings $I_{\alpha}: L^{s_{0}} \rightarrow L^{q_{0}}$ and $I_{\alpha}: L^{s_{1}} \rightarrow L^{q_{1}}$ with

$$
\frac{1}{s_{i}}=\frac{1}{q_{i}}+\frac{\alpha}{n}, \quad i=0,1
$$

are bounded. Let $\frac{1}{\theta}=\frac{1}{s_{0}}-\frac{1}{s_{1}}=\frac{1}{q_{0}}-\frac{1}{q_{1}}$. Furthermore, as

$$
\frac{1}{q_{1}}+\frac{\alpha}{n}=\frac{1}{s_{1}}<\frac{1}{q_{X}} \leq \frac{1}{p_{X}}<\frac{1}{s_{0}}=\frac{1}{q_{0}}+\frac{\alpha}{n},
$$

(4.6) and (4.7) are fulfilled for the interpolation $\left(L^{q_{0}}, L^{q_{1}}\right)_{\theta, s_{0}, X}$. Theorem 4.2 and Corollary 4.3 yield

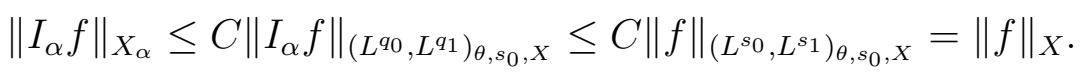

Next, we have with the mapping properties of the convolution operators on r.i.q.B.f.s.

Theorem 5.2. Let $1<p<\infty$ and $X$ be a r.i.q.B.f.s. If $1<p_{X} \leq q_{X}<p^{\prime}$ and $f \in L^{p}$, then we have

$$
\|f * g\|_{X_{\frac{n}{p^{\prime}}}} \leq C\|f\|_{L_{p, \infty}}\|g\|_{X}
$$

for some $C>0$ independent of $f$ and $g$.

Proof. For any $f \in L_{p, \infty}$, write $T_{f}(g)=f * g$. According to the Young inequality, $T_{f}: L^{q} \rightarrow L^{s}$ is bounded when

$$
\frac{1}{p}+\frac{1}{q}=\frac{1}{s}+1
$$

Moreover, we have

$$
\|f * g\|_{L^{s}} \leq C_{p, q}\|f\|_{L_{p, \infty}}\|g\|_{L^{q}}
$$

for some $C_{p, q}>0$ independent of $f$ and $g$.

We first select $q_{0}$ and $q_{1}$ satisfying

$$
1<q_{0}<p_{X} \leq q_{X}<q_{1}<p^{\prime}
$$

and

$$
\frac{1}{q_{0}}-\frac{1}{p^{\prime}}>\frac{1}{p_{X}}
$$

Since

we have $s_{0}, s_{1}$ fulfilling

$$
\frac{1}{q_{1}}>\frac{1}{p^{\prime}} \quad \text { and } \quad \frac{1}{q_{0}}-\frac{1}{p^{\prime}}>\frac{1}{p_{X}}
$$

$$
q_{0}<s_{0}<p_{X} \leq q_{X}<q_{1}<s_{1}
$$

and

$$
\frac{1}{q_{0}}-\frac{1}{s_{0}}=\frac{1}{q_{1}}-\frac{1}{s_{1}}=\frac{1}{p^{\prime}}=1-\frac{1}{p} .
$$

Thus, we find that

$$
\left\|T_{f}(g)\right\|_{L^{s_{i}}} \leq C_{p, q_{i}}\|f\|_{L_{p, \infty}}\|g\|_{L^{q_{i}}}, \quad i=0,1 .
$$

We apply the interpolation functor $(\cdot, \cdot)_{\theta, q_{0}, X}$ on $T_{f}$ with

$$
\frac{1}{\theta}=\frac{1}{s_{0}}-\frac{1}{s_{1}}=\frac{1}{q_{0}}-\frac{1}{q_{1}} .
$$


We have the last identity in view of (5.3). Therefore, $T_{f}:\left(L^{q_{0}}, L^{q_{1}}\right)_{\theta, q_{0}, X} \rightarrow$ $\left(L^{s_{0}}, L^{s_{1}}\right)_{\theta, q_{0}, X}$ is bounded. Since $q_{0}<p_{X} \leq q_{X}<q_{1}$, Corollary 4.3 yields

$$
\left(L^{q_{0}}, L^{q_{1}}\right)_{\theta, q_{0}, X}=X .
$$

Next, we apply the functor $(\cdot, \cdot)_{\theta, q_{0}, X}$ to $L^{s_{0}}$ and $L^{s_{1}}$. Thus, we need to show that (4.7) is satisfied. With respect to the notation used in Theorem 4.2, we have $r=q_{0}$ and

$$
\frac{\alpha}{n}=\frac{1}{q_{0}}-\frac{1}{s_{0}}
$$

By using (5.3)-(5.6), we have

$$
\frac{1}{s_{1}}+\frac{\alpha}{n}=\frac{1}{s_{1}}+\frac{1}{q_{0}}-\frac{1}{s_{0}}=\frac{1}{q_{0}}-\frac{1}{q_{0}}+\frac{1}{q_{1}}=\frac{1}{q_{1}}<\frac{1}{q_{X}} .
$$

Similarly,

$$
\frac{1}{s_{0}}+\frac{\alpha}{n}=\frac{1}{s_{0}}+\frac{1}{q_{0}}-\frac{1}{s_{0}}=\frac{1}{q_{0}}>\frac{1}{p_{X}} .
$$

Thus, condition (4.7) is fulfilled and we are allowed to apply Theorem 4.2 to obtain

$$
\left(L^{s_{0}}, L^{s_{1}}\right)_{\theta, q_{0}, X}=X_{\alpha}
$$

According to (5.3) and (5.6), we find that

$$
\frac{\alpha}{n}=\frac{1}{q_{0}}-\frac{1}{s_{0}}=\frac{1}{p^{\prime}}
$$

That is, $X_{\alpha}=X_{\frac{n}{p^{\prime}}}$. Therefore, $T_{f}: X \rightarrow X_{\frac{n}{p^{\prime}}}$ is bounded. In view of (4.3) and (4.4), we have

$$
\|f * g\|_{X_{\frac{n}{p^{\prime}}}} \leq C\|f\|_{L_{p, \infty}}\|g\|_{X}
$$

for some $C>0$ independent of $f$ and $g$.

When $X$ is the Lebesgue spaces, (5.1) is also named as the Young inequality. Therefore, (5.1) is also a generalization of Young's inequalities to r.i.q.B.f.s. In fact, Corollary 5.1 is a consequence of Theorem 5.2 because $f_{\alpha} \in L_{\frac{n}{n-\alpha}, \infty}$ where $f_{\alpha}(x)=$ $|x|^{n-\alpha}, 0<\alpha<n$.

At the end of this section, we establish the mapping properties of the Fourier integral operators on r.i.q.B.f.s. We recall some basic idea for defining the Fourier integral operators from [31, Chapter IX, Section 3].

Let $m \in \mathbf{R}$. The class $S^{m}$ consists of those infinitely differentiable function $a(x, \xi): \mathbf{R}^{n} \times \mathbf{R}^{n} \rightarrow \mathbf{R}$ satisfying

$$
\left|\partial_{x}^{\tau} \partial_{\xi}^{\gamma} a(x, \xi)\right| \leq A_{\gamma, \tau}(1+|\xi|)^{m-|\gamma|},
$$

for all multi-indices $\gamma, \tau$. The Fourier integral operator $T_{a}$ associated with the symbol $a \in S^{m}$ is given by

$$
\left(T_{a} f\right)(x)=\int_{\mathbf{R}^{n}} e^{2 \pi i \phi(x, \xi)} a(x, \xi) \hat{f}(\xi) d \xi
$$

where the phase function $\phi: \mathbf{R}^{n} \times \mathbf{R}^{n} \rightarrow \mathbf{R}$ is real-valued, homogeneous of degree 1 in $\xi$, and smooth in $(x, \xi)$ when $\xi \neq 0$, on the support of $a$. It is also assumed that $\phi$ satisfies the non-degeneracy condition

$$
\operatorname{det}\left(\frac{\partial^{2} \phi}{\partial x_{i} \partial \xi_{j}}\right) \neq 0
$$


on the support of $a$. For details of the Fourier integral operators, the reader is referred to [31, Chapter IX, Section 3].

Corollary 5.3. Let $T_{a}$ be a Fourier integral operator with $a \in S^{m},-\frac{n}{2}<m<0$. If $X$ is a r.i.q.B.f.s. with $p<p_{X} \leq q_{X}<2$ where

$$
\frac{1}{p}=\frac{1}{2}-\frac{m}{n}
$$

Then, we have

$$
\left\|T_{a} f\right\|_{X_{-m}} \leq C\|f\|_{X}
$$

for some $C>0$ independent of $f$.

Proof. According to [31, Proposition 3.1.4], the Fourier integral operator $T$ satisfies

$$
\left\|T_{a} f\right\|_{L^{2}} \leq C\|f\|_{L^{p}} \quad \text { and } \quad\left\|T_{a} f\right\|_{L^{q}} \leq C\|f\|_{L^{2}}
$$

for some $C>0$, where

$$
\frac{1}{q}=\frac{1}{2}+\frac{m}{n}
$$

We apply the interpolation functor $(\cdot, \cdot)_{-\frac{m}{n}, p, X}$ on $T_{a}$. According to (5.8), Corollary 4.3 yields

$$
\left(L^{p}, L^{2}\right)_{-\frac{m}{n}, p, X}=X .
$$

In view of (5.8) and (5.10), we have

$$
\frac{1}{q}-\frac{m}{n}=\frac{1}{2}<\frac{1}{q_{X}} \leq \frac{1}{p_{X}}<\frac{1}{p}=\frac{1}{2}-\frac{m}{n} .
$$

The above inequalities and (5.10) guarantee that we can apply Theorem 4.2 to the interpolation $\left(L^{2}, L^{q}\right)_{-\frac{m}{n}, p, X}$. Identity (5.8) shows that $\alpha=-m$. Thus, Theorem 4.2 assures that

$$
\left(L^{2}, L^{q}\right)_{-\frac{m}{n}, p, X}=X_{-m}
$$

Hence, we obtain (5.9).

\section{Fourier transform, oscillatory integral operator and restriction theorem}

In this section, we give the main results corresponding to the family $\left\{\hat{X}_{\beta}\right\}$. We extend the mapping properties of the Fourier transform and the oscillatory integral operators to r.i.q.B.f.s. in this section. At the end of this section, we also present the restriction theorem of Fourier transform on r.i.q.B.f.s. as an application of the mapping properties of the oscillatory integral operators.

We present the mapping property for Fourier transform on r.i.q.B.f.s. in the following.

Theorem 6.1. Let $X$ be a r.i.q.B.f.s. with $1<p_{X} \leq q_{X}<2$. Then

$$
\|\hat{f}\|_{\hat{X}} \leq C\|f\|_{X}
$$

for some $C>0$. 
Fourier integrals and Sobolev embedding on rearrangement-invariant quasi-Banach function spaces 911

Proof. We have

$$
\|\hat{f}\|_{L^{\infty}} \leq C\|f\|_{L^{1}}, \quad\|\hat{f}\|_{L^{2}}=\|f\|_{L^{2}} .
$$

We apply the functor $(\cdot, \cdot)_{2,1, X}$ on the Fourier transform. Corollary 4.3 gives

$$
\left(L^{1}, L^{2}\right)_{2,1, X}=X .
$$

By the definition of $K$-functional and the Holmstedt formula, we find that

$$
K\left(\hat{f}, t, L^{\infty}, L^{2}\right)=t K\left(\hat{f}, t^{-1}, L^{2}, L^{\infty}\right) \approx t\left(\int_{0}^{t^{-2}}\left(\hat{f}^{*}(s)\right)^{2} d s\right)^{\frac{1}{2}}
$$

Thus, the fact that $\hat{f}^{*}$ is non-increasing yields

$$
\begin{aligned}
\rho_{X}\left(t^{-1} K\left(\hat{f}, t^{\frac{1}{2}}, L^{\infty}, L^{2}\right)\right) & \geq C \rho_{X}\left(t^{-\frac{1}{2}}\left(\int_{0}^{t^{-1}}\left(\hat{f}^{*}(s)\right)^{2} d s\right)^{\frac{1}{2}}\right) \\
& \geq C \rho_{X}\left(t^{-1} \hat{f}^{*}\left(t^{-1}\right)\right)=\|\hat{f}\|_{\hat{X}}
\end{aligned}
$$

That is, we have the embedding $\left(L^{\infty}, L^{2}\right)_{2,1, X} \hookrightarrow \hat{X}$. In conclusion, the action of the functor $(\cdot, \cdot)_{2,1, X}$ on the Fourier transform establishes (6.1).

The above mapping property for the Fourier transform is one of the main reason why we consider r.i.q.B.f.s. instead of only r.i.B.f.s. in this paper.

When $X=L^{p}$ with $1<p<2$, according to (3.2) and Theorem 6.1, we obtain

$$
\|\hat{f}\|_{L_{p^{\prime}, p}} \leq C\|f\|_{L^{p}}
$$

By using the embedding of Lorentz space [3, Chapter 4, Proposition 4.2]

$$
L_{p^{\prime}, q} \hookrightarrow L_{p^{\prime}, r}, \quad 0<p \leq \infty, \quad \text { and } \quad 0<q<r \leq \infty,
$$

we recover the classical Hausdorff-Young inequality

$$
\|\hat{f}\|_{L^{p^{\prime}}} \leq C\|f\|_{L^{p}}
$$

because $p^{\prime}>p$ when $1<p<2$.

Next, we study a generalization of the Fourier transform, the oscillatory integral operator. The oscillatory integral operator associated with $a(x, y) \in C_{0}^{\infty}\left(\mathbf{R}^{n} \times \mathbf{R}^{n}\right)$ and $\phi \in C^{\infty}\left(\mathbf{R}^{n} \times \mathbf{R}^{n}\right)$ is given by

$$
\left(T_{\lambda} f\right)(x)=\int_{\mathbf{R}^{n}} e^{i \lambda \phi(x, y)} a(x, y) f(x) d y, \quad \lambda>0 .
$$

We call $\phi$ the phase function. For the details of the study of the oscillatory integral operator, the reader is referred to [30, Chapter 2] and [31, Chapter IX].

Theorem 6.2. Let $X$ be a r.i.q.B.f.s. with $1<p_{X} \leq q_{X}<2$. If $\phi$ satisfies the non-degeneracy condition

$$
\operatorname{det}\left(\frac{\partial^{2} \phi}{\partial x_{i} \partial y_{j}}\right) \neq 0
$$

on the support of $a$. Then, for any $\lambda, \epsilon>0$, there exists a $C_{\epsilon}>0$ independent of $\lambda$,

$$
\left\|T_{\lambda} f\right\|_{\hat{X}} \leq C_{\epsilon} \lambda^{-n} \max \left(\lambda^{\frac{n}{p_{X}}-\epsilon}, \lambda^{\frac{n}{q_{X}}+\epsilon}\right)\|f\|_{X}, \quad \forall f \in X .
$$


Proof. According to [30, Theorem 2.1.1], we have

$$
\left\|T_{\lambda} f\right\|_{L^{\infty}} \leq K\|f\|_{L^{1}}
$$

for some $K>0$. Moreover, as $\phi$ satisfies the non-degeneracy condition (6.3) on the support of $a$, we also have

$$
\left\|T_{\lambda} f\right\|_{L^{2}} \leq K \lambda^{-\frac{n}{2}}\|f\|_{L^{2}}
$$

Applying the functor $(\cdot, \cdot)_{2,1, X}$ on $T_{\lambda}$, Corollary 4.3 gives $\left(L^{1}, L^{2}\right)_{2,1, X}=X$. The proof of Theorem 6.1 yields $\left(L^{\infty}, L^{2}\right)_{2,1, X} \hookrightarrow \hat{X}$.

Finally, (4.4) with $M_{0}=K$ and $M_{1}=K \lambda^{-\frac{n}{2}}$ guarantees that for any $\lambda, \epsilon>0$, there exists a $C_{\epsilon}>0$ independent of $\lambda$,

$$
\left\|T_{\lambda} f\right\|_{\hat{X}} \leq C_{\epsilon} \lambda^{-n} \max \left(\lambda^{\frac{n}{p_{X}}-\epsilon} \lambda^{\frac{n}{q_{X}}+\epsilon}\right)\|f\|_{X} .
$$

The above result is an extension of the mapping property for Fourier transform on $X$. On the other hand, some important applications of the boundedness of the oscillatory integral operators lies in the case where the phase function does not satisfy the non-degeneracy condition (6.3). Some oscillatory integral operators of this type are of the form

$$
\left(T_{\lambda} f\right)(z)=\int_{\mathbf{R}^{n-1}} e^{i \lambda \phi(z, y)} a(z, y) f(y) d y, \quad \lambda>0,
$$

where $a \in C_{0}^{\infty}\left(\mathbf{R}^{n} \times \mathbf{R}^{n-1}\right)$ and $\phi: \mathbf{R}^{n} \times \mathbf{R}^{n-1} \rightarrow \mathbf{R}$ is a real $C^{\infty}$ phase function in a neighborhood of $\operatorname{supp} a$.

In this situation we assume that $\phi$ fulfills the Carleson-Sjölin condition. More precisely, the Carleson-Sjölin condition assumes that $\phi$ satisfies

$$
\operatorname{rank}\left(\frac{\partial^{2} \phi}{\partial y_{i} \partial z_{j}}\right) \equiv n-1
$$

and

$$
S_{z_{0}}=\Pi_{T_{z_{0}}^{*} \mathbf{R}^{n}}\left(\mathcal{C}_{\phi}\right)
$$

has everywhere non-vanishing Gaussian curvature on $T_{z_{0}}^{*} \mathbf{R}^{n}$, where $T_{z_{0}}^{*} \mathbf{R}^{n}$ is the vector space of all cotangent vector at $z_{0}$ on $\mathbf{R}^{n}$,

$$
\mathcal{C}_{\phi}=\left\{\left(z, \phi_{z}^{\prime}(z, y), y,-\phi_{y}^{\prime}(z, y)\right):(z, y) \in \mathbf{R}^{n} \times \mathbf{R}^{n-1}\right\}
$$

and $\Pi_{T_{z_{0}}^{*} \mathbf{R}^{n}}: \mathcal{C}_{\phi} \rightarrow T_{z_{0}}^{*} \mathbf{R}^{n}$ is the natural projection.

Obviously, $T_{\lambda}$ maps functions defined on $\mathbf{R}^{n-1}$ to functions on $\mathbf{R}^{n}$. Thus, for any r.i.q.B.f.s. $X$ defined on $\mathbf{R}^{n-1}$, we have to introduce the corresponding r.i.q.B.f.s. on $\mathbf{R}^{n}$.

Let $X$ be a r.i.q.B.f.s. on $\mathbf{R}^{n-1}$. The set $X\left(\mathbf{R}^{n}\right)$ consists of those $f \in \mathcal{M}\left(\mathbf{R}^{n}\right)$ such that

$$
\|f\|_{X\left(\mathbf{R}^{n}\right)}=\rho_{X}\left(f^{*}\right)<\infty .
$$

It is easy to show that whenever $X$ is a r.i.q.B.f.s., $X\left(\mathbf{R}^{n}\right)$ is also a r.i.q.B.f.s.

Theorem 6.3. Let $X$ be a r.i.q.B.f.s. on $\mathbf{R}^{n-1}$ with $1<p_{X} \leq q_{X}<2$. If $\phi$ satisfies the Carleson-Sjölin condition. Then, for any $\epsilon>0$, there exists a constant $C_{\epsilon}>0$ independent of $\lambda>0$ such that

$$
\left\|T_{\lambda} f\right\|_{\hat{X}_{\frac{n+1}{n-1}}\left(\mathbf{R}^{n}\right)} \leq C_{\epsilon} \Lambda(\lambda)\|f\|_{X}
$$

where

$$
\Lambda(\lambda)=\lambda^{-\frac{n(n-1)}{n+1}} \max \left(\lambda^{-\frac{n(n-1)}{p_{X}(n+1)}+\epsilon}, \lambda^{-\frac{n(n-1)}{q_{X}(n+1)}-\epsilon}\right) .
$$


Proof. From [30, Theorem 2.2.1] and [31, Chapter IX, Section 1.2, Theorem 1], we have

$$
\left\|T_{\lambda} f\right\|_{L^{q}\left(\mathbf{R}^{n}\right)} \leq A \lambda^{-n / q}\|f\|_{L^{p}\left(\mathbf{R}^{n-1}\right)}
$$

where

$$
q=\left(\frac{n+1}{n-1}\right) p^{\prime}, \quad \text { and } \quad 1 \leq p \leq 2 .
$$

We apply the interpolation functor $(\cdot, \cdot)_{2,1, X}$ for the operator $T_{\lambda}$. In view of Corollary 4.3 , we find that $\left(L^{1}, L^{2}\right)_{2,1, X}=X$.

Next, we consider $\left(L^{\infty}, L^{2\left(\frac{n+1}{n-1}\right)}\right)_{2,1, X}$. Similar to $(6.2)$, we obtain

$$
\begin{aligned}
K\left(T_{\lambda} f, t, L^{\infty}\left(\mathbf{R}^{n}\right), L^{2\left(\frac{n+1}{n-1}\right)}\left(\mathbf{R}^{n}\right)\right) & =t K\left(T_{\lambda} f, t^{-1}, L^{2\left(\frac{n+1}{n-1}\right)}\left(\mathbf{R}^{n}\right), L^{\infty}\left(\mathbf{R}^{n}\right)\right) \\
& \approx t\left(\int_{0}^{t^{-2\left(\frac{n+1}{n-1}\right)}}\left(\left(T_{\lambda} f\right)^{*}(s)\right)^{2\left(\frac{n+1}{n-1}\right)} d s\right)^{\frac{1}{2\left(\frac{n+1}{n-1}\right)}} .
\end{aligned}
$$

Thus,

$$
\begin{aligned}
\left\|T_{\lambda} f\right\|_{\left(L^{\infty}\left(\mathbf{R}^{n}\right), L^{2\left(\frac{n+1}{n-1}\right)}\left(\mathbf{R}^{n}\right)\right)_{2,1, X}} & =\rho_{X}\left(t^{-1} K\left(T_{\lambda} f, t^{\frac{1}{2}}, L^{\infty}\left(\mathbf{R}^{n}\right), L^{2\left(\frac{n+1}{n-1}\right)}\left(\mathbf{R}^{n}\right)\right)\right) \\
& \geq C \rho_{X}\left(t^{-\frac{1}{2}}\left(\int_{0}^{t^{-\left(\frac{n+1}{n-1}\right)}}\left(\left(T_{\lambda} f\right)^{*}(s)\right)^{2\left(\frac{n+1}{n-1}\right)} d s\right)^{\frac{1}{2\left(\frac{n+1}{n-1}\right)}}\right) \\
& \geq C \rho_{X}\left(t^{-1}\left(T_{\lambda} f\right)^{*}\left(t^{-\frac{n+1}{n-1}}\right)\right)=C\left\|T_{\lambda} f\right\|_{\hat{X}_{\frac{n+1}{n-1}}\left(\mathbf{R}^{n}\right)}
\end{aligned}
$$

for some $C>0$. Hence, we establish (6.7). Finally, (6.8) follows from (4.4) with $M_{0}=A$ and $M_{1}=A \lambda^{-\frac{n(n-1)}{2(n+1)}}$.

Notice that when $X$ is the Lebesgue space $L^{p}$, the epsilon $\epsilon$ in (6.4) and (6.8) can be taken to be zero because $\left\|D_{s}\right\|_{L^{p} \rightarrow L^{p}}=C s^{-1 / p}$ for some $C>0$. Therefore, (6.4) becomes [30, Corollary 2.1.2] and (6.8) reduces to $\Lambda(\lambda)=\lambda^{-n / q}$.

As an important consequence of the mapping properties of the oscillatory integral operators, we present the restriction theorem for the Fourier transform on r.i.q.B.f.s. For the study of the Fourier restriction theorem on $L^{p}$, the reader may consult [30, Corollary 2.2.2] and [31, Chapter IX, Proposition 2.1].

Suppose that $S \subset \mathbf{R}^{n}, n \geq 2$, is a $C^{\infty}$ hypersurface with non-vanishing Gaussian curvature.

Theorem 6.4. Let $S_{0}$ be a compact subset of $S$. Let $X$ be a r.i.q.B.f.s. on $\mathbf{R}^{n}$ with $1<p_{X} \leq q_{X}<\frac{2(n+1)}{n+3}$. Then,

$$
\left\|\hat{f} \chi_{S_{0}}\right\|_{\hat{X}_{\frac{n-1}{n+1}}} \leq C\|f\|_{X}, \quad \forall f \in \mathcal{S}\left(\mathbf{R}^{n}\right) .
$$

Proof. By [30, Corollary 2.2.2] and [31, Chapter IX, Proposition 2.1],we have

$$
\left\|\hat{f} \chi_{S_{0}}\right\|_{L^{r}} \leq C\|f\|_{L^{p}}, \quad \forall f \in \mathcal{S}\left(\mathbf{R}^{n}\right)
$$

where $r=\frac{n-1}{n+1} p^{\prime}$ and $1 \leq p \leq \frac{2(n+1)}{n+3}$. We apply the interpolation functor $(\cdot, \cdot)_{\frac{2 n+2}{n-1}, 1, X}$ for the mapping $f \rightarrow \hat{f} \chi_{S_{0}}$. Corollary 4.3 offers $\left(L^{1}, L^{\frac{2(n+1)}{n+3}}\right)_{\frac{2 n+2}{n-1}, 1, X}=X$. 
Let $\theta=\frac{2(n+1)}{n-1}$. Then, $\frac{1}{\theta}=1-\frac{1}{\frac{2(n+1)}{n+3}}$. Similar to the proof of Theorem 6.3, we find that for any $g \in L^{2}+L^{\infty}$

$$
t^{-1} K\left(g, t^{\frac{1}{\theta}}, L^{\infty}, L^{2}\right)=t^{-\frac{1}{\theta^{\prime}}}\left(\int_{0}^{t^{-\frac{2}{\theta}}}\left(g^{*}(s)\right)^{2} d s\right)^{\frac{1}{2}} .
$$

Consequently,

$$
\rho_{X}\left(t^{-1} K\left(g, t^{\frac{1}{\theta}}, L^{\infty}, L^{2}\right)\right) \geq \rho_{X}\left(t^{-1} g^{*}\left(t^{-\frac{n-1}{n+1}}\right)\right) .
$$

Hence, the embedding $\left(L^{\infty}, L^{2}\right)_{\frac{2 n+2}{n-1}, 1, X} \hookrightarrow \hat{X}_{\frac{n-1}{n+1}}$ is valid and the mapping property (6.9) is established.

\section{Sobolev spaces}

In this section, we establish the Sobolev embedding theorem for Sobolev spaces built on r.i.q.B.f.s. For the details of the Sobolev embedding theorem of the classical Sobolev spaces, the reader may consult [2, Chapter 4]. We first give the definition of the Sobolev spaces associated with r.i.q.B.f.s.

Definition 7.1. Let $k \in \mathbf{N}$ and $X$ be a r.i.q.B.f.s. The Sobolev space $W_{k}^{X}$ consists of those Lebesgue measurable functions $f$ on $\mathbf{R}^{n}$ such that

$$
\|f\|_{W_{k}^{X}}=\sum_{|\gamma| \leq k}\left\|D^{\gamma} f\right\|_{X}<\infty
$$

where $\gamma=\left(\gamma_{1}, \cdots, \gamma_{n}\right)$ is a multi-index and $D^{\gamma} f$ is the distributional derivative of $f$. Write

$$
\mathcal{D}_{k} f=\sum_{|\gamma| \leq k}\left|D^{\gamma} f\right|
$$

In order to match with the classical notion for Sobolev spaces, write $W_{k}^{p}$ for $W_{k}^{X}$ when $X=L^{p}, 1 \leq p \leq \infty$. The following formula represents the $K$-functional of Sobolev spaces in terms of $\mathcal{D}_{k}$ and Lebesgue spaces.

Proposition 7.1. Let $k \in \mathbf{N}$ and $1<p<q<\infty$. We have

$$
K\left(f, t, W_{k}^{p}, W_{k}^{q}\right) \approx K\left(\mathcal{D}_{k} f, t, L^{p}, L^{q}\right) .
$$

Proof. In view of [3, Chapter 5, Corollary 5.13], we have

$$
W_{k}^{p}=\left(W_{k}^{1}, W_{k}^{\infty}\right)_{\frac{1}{p^{\prime}}, p}
$$

where $(\cdot, \cdot)_{\frac{1}{p^{\prime}}, p}$ is the Marcinkiewicz real interpolation functor.

We apply the Holmstedt formula for the $K$-functional for Marcinkiewicz real interpolation functor [3, Chapter 5, Theorem 2.1] to (7.3) and obtain

$$
\begin{aligned}
& K\left(f, t, W_{k}^{p}, W_{k}^{q}\right) \\
& \approx\left(\int_{0}^{t^{\frac{1}{\delta}}}\left(s^{-\frac{1}{p^{\prime}}} K\left(f, t, W_{k}^{1}, W_{k}^{\infty}\right)\right)^{p} \frac{d s}{s}\right)^{\frac{1}{p}}+t^{\frac{1}{\delta}}\left(\int_{t^{\frac{1}{\delta}}}^{\infty}\left(s^{-\frac{1}{q^{\gamma}}} K\left(f, t, W_{k}^{1}, W_{k}^{\infty}\right)\right)^{q} \frac{d s}{s}\right)^{\frac{1}{q}} .
\end{aligned}
$$

The $K$-functional of Sobolev spaces [8] is given by

$$
K\left(f, t, W_{k}^{1}, W_{k}^{\infty}\right) \approx \int_{0}^{t}\left(\mathcal{D}_{k} f\right)^{*}(s) d s .
$$


Fourier integrals and Sobolev embedding on rearrangement-invariant quasi-Banach function spaces 915

Therefore,

$$
\begin{aligned}
& K\left(f, t, W_{k}^{p}, W_{k}^{q}\right) \\
& \approx\left(\int_{0}^{t^{\frac{1}{\delta}}}\left(s^{-1} \int_{0}^{s}\left(\mathcal{D}_{k} f\right)^{*}(u) d u\right)^{p} d s\right)^{\frac{1}{p}}+t^{\frac{1}{\delta}}\left(\int_{t^{\frac{1}{\delta}}}^{\infty}\left(s^{-1} \int_{0}^{s}\left(\mathcal{D}_{k} f\right)^{*}(u) d u\right)^{q} d s\right)^{\frac{1}{q}}
\end{aligned}
$$

where $\delta=\frac{1}{p}-\frac{1}{q}$.

By applying Hardy's inequality [10, Theorem 2.2.1] on the intervals $\left(0, t^{\frac{1}{\delta}}\right)$ and $\left(t^{\delta}, \infty\right)$, the Holmstedt formulas for the $K$-functionals of Lebesgue spaces [22] assure that

$$
\begin{aligned}
K\left(f, t, W_{k}^{p}, W_{k}^{q}\right) & \leq C\left(\left(\int_{0}^{t^{\frac{1}{\delta}}}\left(\left(\mathcal{D}_{k} f\right)^{*}(s)\right)^{p} d s\right)^{\frac{1}{p}}+t^{\frac{1}{\delta}}\left(\int_{t^{\frac{1}{\delta}}}^{\infty}\left(\left(\mathcal{D}_{k} f\right)^{*}(s)\right)^{q} d s\right)^{\frac{1}{q}}\right) \\
& \leq C K\left(\mathcal{D}_{k} f, t, L^{p}, L^{q}\right)
\end{aligned}
$$

for some $C>0$.

For the reserve inequality, since $\left(\mathcal{D}_{k} f\right)^{*}$ is a non-increasing function, we have,

$$
\begin{aligned}
K\left(f, t, W_{k}^{p}, W_{k}^{q}\right) & \geq C\left(\left(\int_{0}^{t^{\frac{1}{\delta}}}\left(\left(\mathcal{D}_{k} f\right)^{*}(s)\right)^{p} \frac{d s}{s}\right)^{\frac{1}{p}}+t^{\frac{1}{\delta}}\left(\int_{t^{\frac{1}{\delta}}}^{\infty}\left(\left(\mathcal{D}_{k} f\right)^{*}(s)\right)^{q} \frac{d s}{s}\right)^{\frac{1}{q}}\right) \\
& \geq C K\left(\mathcal{D}_{k} f, t, L^{p}, L^{q}\right) .
\end{aligned}
$$

We now give the interpolation results of the Sobolev spaces under the functor $(\cdot, \cdot)_{\theta, r, X} \cdot$

Theorem 7.2. Let $k \in \mathbf{N}, 0 \leq \alpha<\infty$ and $X$ be a r.i.q.B.f.s. with $0<p_{X} \leq$ $q_{X}<\frac{n}{\alpha}$. Suppose $0<p_{0}<p_{X} \leq q_{X}<p_{1}<\infty$ and $r, \theta$ satisfy (4.6) and (4.7). Then,

$$
\left(W_{k}^{p_{0}}, W_{k}^{p_{1}}\right)_{\theta, r, X}=W_{k}^{X_{\alpha}} .
$$

Proof. The definition of $(\cdot, \cdot)_{(\theta, r, X)}$ and $(7.2)$ assures that

$$
\begin{aligned}
\|f\|_{\left(W_{k}^{p_{0}}, W_{k}^{p_{1}}\right)_{\theta, r, X}} & =\rho_{X}\left(t^{-\frac{1}{r}} K\left(f, t^{\frac{1}{\theta}}, W_{k}^{p_{0}}, W_{k}^{p_{1}}\right)\right) \approx \rho_{X}\left(t^{-\frac{1}{r}} K\left(\mathcal{D}_{k} f, t^{\frac{1}{\theta}}, L^{p_{0}}, L^{p_{1}}\right)\right) \\
& =\left\|\mathcal{D}_{k} f\right\|_{\left(L^{p_{0}}, L^{p_{1}}\right)_{\theta, r, X}}=\left\|\mathcal{D}_{k} f\right\|_{X_{\alpha}} .
\end{aligned}
$$

It is obvious that the quasi-norms $\|\cdot\|_{W_{k}^{X_{\alpha}}}$ and $\left\|\mathcal{D}_{k}(\cdot)\right\|_{X_{\alpha}}$ are mutually equivalent. Thus, we obtain (7.4).

We are now apply Theorem 7.2 to establish the main result of this section, the Sobolev embedding theorem for $W_{k}^{X}$.

Theorem 7.3. Let $k \in \mathbf{N}$ and $X$ be a r.i.q.B.f.s. with $0<p_{X} \leq q_{X}<\frac{n}{k}$. We have

$$
W_{k}^{X} \hookrightarrow X_{k} .
$$

Proof. We choose $p_{0}, p_{1}$ such that

$$
0<p_{0}<p_{X} \leq q_{X}<p_{1}<\frac{n}{k} .
$$


The classical Sobolev embedding theorem on Lebesgue spaces assures that

$$
\begin{aligned}
& W_{k}^{p_{0}} \hookrightarrow L^{q_{0}}, \quad \frac{1}{q_{0}}=\frac{1}{p_{0}}-\frac{k}{n}, \\
& W_{k}^{p_{1}} \hookrightarrow L^{q_{1}}, \quad \frac{1}{q_{1}}=\frac{1}{p_{1}}-\frac{k}{n} .
\end{aligned}
$$

We apply the interpolation $(\cdot, \cdot)_{\theta, p_{0}, X}$ on the embedding operator with $\frac{1}{\theta}=\frac{1}{p_{0}}-\frac{1}{p_{1}}$. Theorem 7.2 gives

$$
\left(W_{k}^{p_{0}}, W_{k}^{p_{1}}\right)_{\theta, p_{0}, X}=W_{k}^{X} .
$$

The $q_{0}$ and $q_{1}$ given in (7.7) and (7.8) satisfy

$$
\frac{1}{\theta}=\frac{1}{p_{0}}-\frac{1}{p_{1}}=\frac{1}{q_{0}}-\frac{1}{q_{1}}, \text { and } \quad \frac{1}{p_{0}}=\frac{1}{q_{0}}+\frac{k}{n} .
$$

Therefore, (4.6) is fulfilled for the interpolation $\left(L^{q_{0}}, L^{q_{1}}\right)_{\theta, p_{0}, X}$. Furthermore, we have

$$
\frac{1}{q_{1}}+\frac{k}{n}=\frac{1}{p_{1}}<\frac{1}{q_{X}} \leq \frac{1}{p_{X}}<\frac{1}{p_{0}}=\frac{1}{q_{0}}+\frac{k}{n} .
$$

That is, (4.7) is also satisfied for the interpolation $\left(L^{q_{0}}, L^{q_{1}}\right)_{\theta, p_{0}, X}$. Thus, Theorem 4.2 asserts that

$$
W_{k}^{X}=\left(W_{k}^{p_{0}}, W_{k}^{p_{1}}\right)_{\theta, r, X} \hookrightarrow\left(L^{q_{0}}, L^{q_{1}}\right)_{\theta, p_{0}, X}=X_{k} .
$$

Obviously, the above result generalizes the classical Sobolev embedding theorem for Lebesgue spaces to r.i.q.B.f.s. In the next section, we present some further generalizations of the Sobolev embedding theorem by extending the Sobolev-Jawerth embedding theorem to Triebel-Lizorkin spaces associated with r.i.q.B.f.s.

\section{Triebel-Lizorkin spaces and Hardy spaces}

The Triebel-Lizorkin spaces provide a unified framework for the studies of a number of important function spaces in analysis such as the Lebesgue spaces, the Sobolev spaces and the Hardy spaces [32, 33].

Recently, there are some extensions of the notion of the Triebel-Lizorkin spaces with the Lebesgue spaces replaced by some general function spaces. In [16], a new family of Triebel-Lizorkin spaces is introduced by using the Littlewood-Paley functions and the spectral synthesis and Luzin approximation of this family of function spaces are investigated. Moreover, the smooth atomic and molecular decompositions are obtained in [18, Section 3]. The duality properties of these function spaces are investigated in [21].

Another type of generalization of the Triebel-Lizorkin spaces by using the Peetre's maximal function is introduced in [26]. The main theme of this section is to study the Triebel-Lizorkin spaces associated with r.i.q.B.f.s. Especially, we are interested on the interpolation properties of these function spaces.

Let us recall the definition of the Triebel-Lizorkin spaces associated with r.i.q.B.f.s. via the Littlewood-Paley functions from [18, Definition 2.1]. Let $\mathcal{P}$ denote the class of polynomials in $\mathbf{R}^{n}$.

Definition 8.1. Let $\alpha \in \mathbf{R}, 0<q<\infty$ and $X$ be a r.i.q.B.f.s. with $0<p_{X} \leq$ $q_{X}<\infty$. The Triebel-Lizorkin space $\dot{F}_{q, X}^{\alpha}$ consists of those $f \in \mathcal{S}^{\prime}\left(\mathbf{R}^{n}\right) / \mathcal{P}$ satisfying

$$
\|f\|_{\dot{F}_{q, X}^{\alpha}}=\left\|\left(\sum_{j=-\infty}^{\infty}\left(2^{j \alpha}\left|f * \varphi_{j}\right|\right)^{q}\right)^{\frac{1}{q}}\right\|_{X}<\infty,
$$


Fourier integrals and Sobolev embedding on rearrangement-invariant quasi-Banach function spaces 917

where $\varphi_{j}(x)=2^{j n} \varphi\left(2^{j} x\right), j \in \mathbf{Z}$ and $\varphi \in \mathcal{S}\left(\mathbf{R}^{n}\right)$ satisfy

$$
\begin{aligned}
& \operatorname{supp} \hat{\varphi} \subseteq\left\{\xi \in \mathbf{R}^{n}: 1 / 2 \leq|\xi| \leq 2\right\} \quad \text { and } \\
&|\hat{\varphi}(\xi)| \geq C, \quad 3 / 5 \leq|\xi| \leq 5 / 3
\end{aligned}
$$

for some $C>0$.

We also recall the definition of the corresponding sequence space [18, Definition 2.3]. Let $\mathcal{Q}=\left\{Q_{i, k}: i \in \mathbf{Z}, k \in \mathbf{Z}^{n}\right\}$ denote the set of dyadic cubes, where $Q_{i, k}=\left\{\left(x_{1}, \ldots, x_{n}\right) \in \mathbf{R}^{n}: k_{j} \leq 2^{i} x_{j}<k_{j}+1, j=1, \ldots, n\right\}$ and $k=\left(k_{1}, \ldots, k_{n}\right)$. We denote the Lebesgue measure of $Q \in \mathcal{Q}$ by $|Q|$ and the side length of $Q$ by $l(Q)$.

Definition 8.2. Let $\alpha \in \mathbf{R}, 0<q<\infty$ and $X$ be a r.i.q.B.f.s. with $0<$ $p_{X} \leq q_{X}<\infty$. The Triebel-Lizorkin sequence space $\dot{f}_{q, X}^{\alpha}$ consists of those complex sequence $s=\left\{s_{Q}\right\}_{Q \in \mathcal{Q}}$

$$
\|f\|_{f_{q, X}^{\alpha}}=\left\|\left(\sum_{Q \in \mathcal{Q}}\left(|Q|^{-\alpha / n}\left|s_{Q}\right| \tilde{\chi}_{Q}\right)^{q}\right)^{\frac{1}{q}}\right\|_{X}<\infty
$$

where $\tilde{\chi}_{Q}=|Q|^{-1 / 2} \chi_{Q}$.

When $X=L^{p}, \dot{F}_{q, X}^{\alpha}$ and $\dot{f}_{q, X}^{\alpha}$ becomes the well-known Triebel-Lizorkin space $F_{q, p}^{\alpha}$ and the Triebel-Lizorkin sequence space $f_{q, p}^{\alpha}$, respectively [12, 33]. All results obtained for the Littlewood-Paley spaces in [18] are also valid for $\dot{F}_{q, X}^{\alpha}$. For instance, when $0<p_{X} \leq q_{X}<\infty$, the definition of $\dot{F}_{q, X}^{\alpha}$ is independent of the function $\varphi$ [18, Theorems 3.1 and 4.4].

Additionally, the $\varphi-\psi$ transforms are also bounded. Precisely, the $\varphi-\psi$ transforms consist of two operators $\mathrm{S}_{\varphi}$ and $\mathrm{T}_{\psi}$ generated by a pair of functions $\varphi, \psi \in \mathcal{S}\left(\mathbf{R}^{n}\right)$ satisfying (8.2), (8.3) and

$$
\sum_{j \in \mathbf{Z}} \hat{\varphi}\left(2^{-j} \xi\right) \overline{\hat{\psi}\left(2^{-j} \xi\right)}=1, \quad \xi \neq 0
$$

(see $[12$, p. 45, (2.1)-(2.4)]).

We set $\varphi_{\nu}(x)=2^{\nu n} \varphi\left(2^{\nu} x\right), \psi_{\nu}(x)=2^{\nu n} \psi\left(2^{\nu} x\right)$ and $\varphi_{Q}(x)=|Q|^{-1 / 2} \varphi\left(2^{\nu} x-k\right)$, $\psi_{Q}(x)=|Q|^{-1 / 2} \psi\left(2^{\nu} x-k\right), \nu \in \mathbf{Z}, k \in \mathbf{Z}^{n}$ and $Q=Q_{\nu, k}$. For any $f \in \mathcal{S}^{\prime}\left(\mathbf{R}^{n}\right) / \mathcal{P}$ and for any complex-valued sequence $s=\left\{s_{Q}\right\}_{Q \in \mathcal{Q}}$, we define $\mathrm{S}_{\varphi}(f)=\left\{\left(\mathrm{S}_{\varphi} f\right)_{Q}\right\}_{Q \in \mathcal{Q}}=$ $\left\{\left\langle f, \varphi_{Q}\right\rangle\right\}_{Q \in \mathcal{Q}}$ and $\mathrm{T}_{\psi}(s)=\sum_{Q} s_{Q} \psi_{Q}$. It is well known that $\mathrm{T}_{\psi} \circ \mathrm{S}_{\varphi}=i d$ in $\mathcal{S}^{\prime}\left(\mathbf{R}^{n}\right) / \mathcal{P}$ (see [17, Theorem 6.1]). By [18, Theorems 3.1 and 4.8], when $0<p_{X} \leq q_{X}<\infty$, the operators

$$
\mathrm{S}_{\varphi}: \dot{F}_{q, X}^{\alpha} \rightarrow \dot{f}_{q, X}^{\alpha}, \quad \text { and } \quad \mathrm{T}_{\psi}: \dot{f}_{q, X}^{\alpha} \rightarrow \dot{F}_{q, X}^{\alpha}
$$

are bounded. Furthermore,

$$
\|f\|_{\dot{F}_{q, X}^{\alpha}} \approx\left\|\mathrm{S}_{\varphi}(f)\right\|_{\dot{f}_{q, X}^{\alpha}}
$$

Moreover, $\dot{F}_{q, X}^{\alpha}$ also possesses the atomic decompositions and molecular characterizations [18, Theorems 3.1 and 3.6].

We restate the definitions of $G^{\alpha q}, m^{\alpha q}$ and $A^{\alpha q}$ from [12]. We need these operators to state the formula for the $K$-functional of $\dot{F}_{q, p}^{\alpha}$. 
Definition 8.3. Let $\alpha \in \mathbf{R}$ and $0<q<\infty$. For any complex sequence $s=$ $\left\{s_{Q}\right\}_{Q \in \mathcal{Q}}$ and $Q \in \mathcal{Q}$, define

$$
\begin{aligned}
& G_{Q}^{\alpha q}(s)(x)=\left(\sum_{P \subset Q}\left(|P|^{-\alpha / n}\left|s_{P}\right| \tilde{\chi}_{P}(x)\right)^{q}\right)^{\frac{1}{q}}, \\
& G^{\alpha q}(s)(x)=\left(\sum_{P \in \mathcal{Q}}\left(|P|^{-\alpha / n}\left|s_{P}\right| \tilde{\chi}_{P}(x)\right)^{q}\right)^{\frac{1}{q}} .
\end{aligned}
$$

Set

$$
m_{Q}^{\alpha q}(s)=\inf \left\{\epsilon:\left|\left\{x \in Q: G_{Q}^{\alpha q}(s)(x)>\epsilon\right\}\right|<|Q| / 4\right\} .
$$

We call $m_{Q}^{\alpha q}(s)$ the " $\frac{1}{4}$-median" of $G_{Q}^{\alpha q}(s)$ on $Q$. Let

$$
m^{\alpha q}(s)(x)=\sup _{Q \in \mathcal{Q}} m_{Q}^{\alpha q}(s) \chi_{Q}(x) .
$$

For any $f \in \mathcal{S}^{\prime}\left(\mathbf{R}^{n}\right)$, write

$$
A^{\alpha q} f=m^{\alpha q}\left(\mathrm{~S}_{\varphi} f\right) .
$$

According to the definition of $\dot{f}_{q, X}^{\alpha}$, we have

$$
\left\|G^{\alpha q}(s)\right\|_{X} \approx\|s\|_{\dot{f}_{q, X}^{\alpha}} .
$$

We give an estimate for the $K$-functional of $\dot{F}_{q, X}^{\alpha}$ in the following.

Proposition 8.1. Suppose $\alpha \in \mathbf{R}, 0<q<\infty$ and $0<p_{0}<p_{1}<\infty$. Then,

$$
K\left(f, t, \dot{F}_{q, p_{0}}^{\alpha}, \dot{F}_{q, p_{1}}^{\alpha}\right) \approx K\left(A^{\alpha q} f, t, L^{p_{0}}, L^{p_{1}}\right) .
$$

For the proof of the above result, the reader is referred to [12, Corollary 6.7].

We present the interpolation result for the Triebel-Lizorkin spaces $\dot{F}_{q, p}^{\alpha}$ under the action of functor $(\cdot, \cdot)_{\theta, r, X}$. The proof of the following result follows from the ideas of the proof of [12, Proposition 5.5].

Theorem 8.2. Let $0 \leq \alpha<\infty, \beta \in \mathbf{R}, 0<q<\infty$ and $X$ be a r.i.q.B.f.s. with $0<p_{X} \leq q_{X}<\frac{n}{\alpha}$. Suppose $0<p_{0}<p_{X} \leq q_{X}<p_{1}<\infty$ and $r, \theta$ satisfy (4.6) and (4.7). Then,

Proof. We have

$$
\left(\dot{F}_{q, p_{0}}^{\beta}, \dot{F}_{q, p_{1}}^{\beta}\right)_{\theta, r, X}=\dot{F}_{q, X_{\alpha}}^{\beta} .
$$

$$
\begin{aligned}
\|f\|_{\left(\dot{F}_{q, p_{0}}^{\beta}, \dot{F}_{q, p_{1}}^{\beta}\right)_{\theta, r, X}} & \left.=\rho_{X}\left(t^{-\frac{1}{r}} K\left(f, t^{\frac{1}{\theta}}, \dot{F}_{q, p_{0}}^{\beta}, \dot{F}_{q, p_{1}}^{\beta}\right)\right) \approx \rho_{X}\left(t^{-\frac{1}{r}} K\left(A^{\beta q} f, t^{\frac{1}{\theta}}, L^{p_{0}}, L^{p_{1}}\right)\right)\right) \\
& =\left\|A^{\beta q} f\right\|_{\left(L^{p_{0}}, L^{p_{1}}\right)_{\theta, r, X}} \approx\left\|A^{\beta q} f\right\|_{X_{\alpha}} .
\end{aligned}
$$

Therefore, it remains to show that

$$
\left\|A^{\beta q} f\right\|_{X_{\alpha}} \approx\|f\|_{\dot{F}_{q, X_{\alpha}}^{\beta}} .
$$

Moreover, in view of (8.5) and (8.10), (8.12) follows from

$$
\|s\|_{\dot{F}_{q, X_{\alpha}}^{\beta}} \approx\left\|m^{\beta q}(s)\right\|_{X_{\alpha}} .
$$

To prove (8.13), notice that we have

$$
\left|\left\{x: m^{\beta q}(s)(x)>t\right\}\right| \leq C \|\left\{x: G^{\beta q}(s)(x)>t\right\} \mid, \quad \forall t>0
$$

for some $C>0$, see the proof of [12, Propositin 5.5]. That is,

$$
\left(m^{\beta q}(s)\right)^{*}(t) \leq\left(G^{\beta q}(s)\right)^{*}(t / B)
$$


Fourier integrals and Sobolev embedding on rearrangement-invariant quasi-Banach function spaces 919

for some $B>0$ independent of $t$. Thus,

$$
\begin{aligned}
\left\|m^{\beta q}(s)\right\|_{X_{\alpha}} & =\rho_{X}\left(t^{\alpha / n}\left(m^{\beta q}(s)\right)^{*}(t)\right. \\
& \leq C \rho_{X}\left((t / B)^{-\alpha / n}\left(G^{\beta q}(s)\right)^{*}(t / B)\right) \leq C\left\|G^{\beta q}(s)\right\|_{X_{\alpha}}
\end{aligned}
$$

for some $C>0$. We use the assumption $0<p_{X} \leq q_{X}<\infty$ for the last inequality. Thus, (8.11) gives

$$
\left\|m^{\beta q}(s)\right\|_{X_{\alpha}} \leq C\|s\|_{\dot{F}_{q, X_{\alpha}}^{\beta}} .
$$

To establish the reserve inequality, we recall some notation from [12]. For any $Q \in \mathcal{Q}$, let

$$
E_{Q}=\left\{x \in Q: G_{Q}^{\beta q}(s)(x) \leq m^{\beta q}(s)(x)\right\} .
$$

According to $[12,(5.10)]$, we have $\frac{\left|E_{Q}\right|}{|Q|} \geq \frac{3}{4}$ and

$$
\left(\sum_{Q}\left(|Q|^{-\beta / n}\left|s_{Q}\right| \tilde{\chi}_{E_{Q}}(s)\right)^{q}\right)^{1 / q} \leq C m^{\beta q}(s)(x), \quad \forall x \in \mathbf{R}^{n} .
$$

Since $X$ is a r.i.q.B.f.s. with $0<p_{X} \leq q_{X}<n / \alpha$, (3.1) guarantees that $0<$ $p_{X_{\alpha}} \leq q_{X_{\alpha}}<\infty$. Therefore, the Fefferman-Stein vector-valued maximal inequalities are valid on $X_{\alpha}$ [18, Theorem 4.8]. That is, there exists an $a>0$ such that

$$
\left\|\left(\sum_{k \in \mathbf{N}}\left(\mathrm{M} f_{k}\right)^{q / a}\right)^{a / q}\right\|_{X_{\alpha}^{1 / a}} \leq C\left\|\left(\sum_{k \in \mathbf{N}}\left|f_{k}\right|^{q / a}\right)^{a / q}\right\|_{X_{\alpha}^{1 / a}}
$$

for some $C>0$ where $\mathrm{M} f$ denote the Hardy-Littlewood maximal function of $f$.

Similar to the proof of [12, Proposition 2.7], with the constant $a$ given in (8.15), inequality (8.15) ensures that for any $s=\left\{s_{Q}\right\}_{Q \in \mathcal{Q}}$,

$$
\begin{aligned}
\|s\|_{\dot{F}_{q, X_{\alpha}}^{\beta}} & \leq(4 / 3)^{-1 / a}\left\|\left(\sum_{Q}\left(\mathrm{M}\left(|Q|^{-\beta / n}\left|s_{Q}\right| \tilde{\chi}_{E_{Q}}\right)^{a}\right)^{q / a}\right)^{a / q}\right\|_{X_{\alpha}^{1 / a}}^{1 / a} \\
& \leq C\left\|\left(\sum_{Q}\left(|Q|^{-\beta / n}\left|s_{Q}\right| \tilde{\chi}_{E_{Q}}\right)^{q}\right)^{\frac{1}{q}}\right\|_{X_{\alpha}} .
\end{aligned}
$$

Thus, inequalities (8.14) and (8.16) assert that

$$
\|s\|_{\dot{F}_{q, X_{\alpha}}^{\beta}} \leq C\left\|m^{\beta q}(s)\right\|_{X_{\alpha}} .
$$

Finally, (8.12) and (8.13) are valid and hence, we establish our promised interpolation results.

Let $X$ be a r.i.q.B.f.s. In view of the Littlewood-Paley characterization of r.i.q. B.f.s. [18, Theorem 4.10], whenever $1<p_{X} \leq q_{X}<\infty, X=\dot{F}_{2, X}^{0}$. Therefore, the above theorem is a generalization of Theorem 4.2 .

Similarly, the following theorem is an extension of the Sobolev embedding of $W_{k}^{X}$. Precisely, we have the Sobolev-Jawerth embedding of $\dot{F}_{q, X}^{\alpha}$ which is an extension of the classical Sobolev-Jawerth embedding [23, 32].

Theorem 8.3. Let $-\infty<\beta_{1} \leq \beta_{0}<\infty, 0<u, v<\infty$. Suppose that

$$
\alpha=\beta_{0}-\beta_{1} \text {. }
$$


If $X$ is a r.i.q.B.f.s. with $0<p_{X} \leq q_{X}<\frac{n}{\alpha}$, then

$$
\dot{F}_{u, X}^{\beta_{0}} \hookrightarrow \dot{F}_{v, X_{\alpha}}^{\beta_{1}}
$$

Proof. Select $p_{0}, p_{1}$ such that

$$
0<p_{0}<p_{X} \leq q_{X}<p_{1}<\frac{n}{\alpha} .
$$

By [33, Theorem 2.7.1], we have the following embedding

$$
\begin{aligned}
& \dot{F}_{u, p_{0}}^{\beta_{0}} \hookrightarrow \dot{F}_{v, q_{0}}^{\beta_{1}}, \quad \text { where } \frac{n}{q_{0}}=\frac{n}{p_{0}}-\beta_{0}+\beta_{1}, \\
& \dot{F}_{u, p_{1}}^{\beta_{0}} \hookrightarrow \dot{F}_{v, q_{1}}^{\beta_{1}}, \quad \text { where } \frac{n}{q_{1}}=\frac{n}{p_{1}}-\beta_{0}+\beta_{1} .
\end{aligned}
$$

We apply the interpolation $(\cdot, \cdot)_{\theta, p_{0}, X}$ on the embedding operator with $\frac{1}{\theta}=\frac{1}{p_{0}}-\frac{1}{p_{1}}$. Theorem 8.2 assures that

$$
\left(\dot{F}_{u, p_{0}}^{\beta_{0}}, \dot{F}_{u, p_{1}}^{\beta_{0}}\right)_{\theta, p_{0}, X}=\dot{F}_{u, X}^{\beta_{0}} .
$$

Next, we deal with the interpolation $\left(\dot{F}_{v, q_{0}}^{\beta_{1}}, \dot{F}_{v, q_{1}}^{\beta_{1}}\right)_{\theta, p_{0}, X}$. From the conditions imposed on $q_{0}$ and $q_{1}$ in (8.19) and (8.20), we find that

$$
\frac{1}{\theta}=\frac{1}{p_{0}}-\frac{1}{p_{1}}=\frac{1}{q_{0}}-\frac{1}{q_{1}}
$$

Moreover,

$$
\frac{1}{p_{0}}=\frac{1}{q_{0}}+\frac{\beta_{0}-\beta_{1}}{n}=\frac{1}{q_{0}}+\frac{\alpha}{n}
$$

Therefore, (4.6) is satisfied for the interpolation $\left(\dot{F}_{v, q_{0}}^{\beta_{1}}, \dot{F}_{v, q_{1}}^{\beta_{1}}\right)_{\theta, p_{0}, X}$.

Furthermore, (8.17), (8.18), (8.19) and (8.20) also guarantee that

$$
\frac{1}{q_{1}}+\frac{\beta_{0}-\beta_{1}}{n}=\frac{1}{p_{1}}<\frac{1}{q_{X}} \leq \frac{1}{p_{X}}<\frac{1}{p_{0}}=\frac{1}{q_{0}}+\frac{\beta_{0}-\beta_{1}}{n} .
$$

Thus, (4.7) is also fulfilled for the interpolation $\left(\dot{F}_{v, q_{0}}^{\beta_{1}}, \dot{F}_{v, q_{1}}^{\beta_{1}}\right)_{\theta, p_{0}, X}$. Hence, Theorem 8.2 yields

$$
\left(\dot{F}_{v, q_{0}}^{\beta_{1}}, \dot{F}_{v, q_{1}}^{\beta_{1}}\right)_{\theta, p_{0}, X}=\dot{F}_{v, X_{\alpha}}^{\beta_{1}} .
$$

In conclusion, the embedding $\dot{F}_{u, X}^{\beta_{0}} \hookrightarrow \dot{F}_{v, X_{\alpha}}^{\beta_{1}}$ is established.

The above results are also valid for the inhomogeneous version of $\dot{F}_{q, X}^{\alpha}$. Notice that the Sobolev spaces $W_{k}^{X}$ is a member of the inhomogeneous version of $\dot{F}_{q, X}^{\alpha}$. For simplicity, we leave the details to the reader.

We apply the above results to another important member of $\dot{F}_{q, X}^{\alpha}$, the Hardy spaces associated with $X$.

Definition 8.4. Let $X$ be a r.i.q.B.f.s. with $0<p_{X} \leq q_{X}<\infty$. The Hardy space associated with $X, H_{X}$, consists of those $f \in \mathcal{S}^{\prime}\left(\mathbf{R}^{n}\right) / \mathcal{P}$ such that

$$
\|f\|_{H_{X}}=\left\|\left(\sum_{j \in \mathbf{Z}}\left|\varphi_{j} * f\right|^{2}\right)^{\frac{1}{2}}\right\|_{X}<\infty
$$

where $\varphi \in \mathcal{S}\left(\mathbf{R}^{n}\right)$ satisfying (8.2) and (8.3). 
In order to match with the notations for the classical Hardy spaces, when $X=L^{p}$ with $0<p \leq 1$, we write $H_{X}$ by $H_{p}$. When $X$ is a Lorentz space, then $H_{X}$ is the Hardy-Lorentz spaces studied in [1]. If $X$ is generated by a growth function of lower type (see [34, p.403]), then $H_{X}$ is the Hardy type Orlicz spaces considered in [34]. The Littlewood-Paley characterization of r.i.q.B.f.s. is given in [18, Theorem 4.10].

Proposition 8.4. Let $X$ be a r.i.q.B.f.s. If $1<p_{X} \leq q_{X}<\infty$, then $H_{X}=X$.

Thus, similar to the Hardy spaces associated with Lebesgue spaces $H_{p}, 0<p \leq 1$, we are particular interested on $H_{X}$ with $0<p_{X} \leq 1$. The following is a special case of Theorem 8.2.

Corollary 8.5. Let $X$ be a r.i.q.B.f.s. with $0<p_{X} \leq q_{X}<\frac{n}{\alpha}$. Suppose $0<$ $p_{0}<p_{X} \leq q_{X}<p_{1}<\infty$ and $r, \theta$ satisfy (4.6) and (4.7). Then,

$$
\left(H_{p_{0}}, H_{p_{1}}\right)_{\theta, r, X}=H_{X_{\alpha}} \text {. }
$$

Let $0<p \leq 1,0<\alpha<n$ and $\frac{1}{q}+\frac{\alpha}{n}=\frac{1}{p}$. From [25], we have

$$
\left\|I_{\alpha}(f)\right\|_{H_{q}} \leq C\|f\|_{H_{p}}, \quad \forall f \in H_{p}
$$

for some $C>0$ By applying Proposition 8.4, Corollary 8.5 and (8.21) on the fractional integral operator $I_{\alpha}$, we establish the following theorem.

Theorem 8.6. Let $0<\alpha<n$ and $X$ be a r.i.q.B.f.s. If $0<p_{X} \leq q_{X}<\infty$, then

$$
\begin{aligned}
\left\|I_{\alpha}(f)\right\|_{H_{X_{\alpha}}} & \leq C\|f\|_{H_{X}}, \quad p_{X} \leq \frac{n}{n+\alpha}, \\
\left\|I_{\alpha}(f)\right\|_{X_{\alpha}} & \leq C\|f\|_{H_{X}}, \quad p_{X}>\frac{n}{n+\alpha}
\end{aligned}
$$

for some $C>0$.

In particular, the above result recovers the mapping properties for the fractional integral operators on Hardy-Lorentz spaces, see [20, Theorem 5.5].

Acknowledgement. The author would like to thank the reviewer for his/her valuable suggestions to improve the presentation of this paper.

\section{References}

[1] Abu-Shammala, W., and A. Torchinsky: The Hardy-Lorentz spaces $H^{p, q}$. Studia Math. 182, 2007, 283-294.

[2] Adams, R., and J. Fournier: Sobolev spaces. - Academic Press, 2003.

[3] Bennett, C., and R. Sharpley: Interpolations of operators. - Academic Press, 1988.

[4] Bergh, J., and J. LÖFström: Interpolation spaces. - Springer-Verlag, 1976.

[5] Brudnyi, Y., and N. KRugluak: Interpolation functors and interpolation spaces. Volume I. - North-Holland, 1991.

[6] Cruz-Uribe, D., J. Martell, and C. PÉrez: Weights, extrapolation and the theory of Rubio de Francia. - Oper. Theory Adv. Appl. 215, Birkhäuser, 2011.

[7] Curbera, G., J. García-Cuerva, J. Martell, and C. PÉrez: Extrapolation with weights, rearrangement-invariant function spaces, modular inequalities and applications to singular integrals. - Adv. Math. 203, 2006, 256-318.

[8] DeVore, R., and K. Scherer: Interpolation of linear operators on Sobolev spaces. - Ann. of Math. 109, 1979, 583-599.

[9] Edmunds, D. E., R. Kerman, and L. PiCK: Optimal Sobolev imbeddings involving rearrangement-invariant quasinorms. - J. Funct. Anal. 170, 2000, 307-355. 
[10] Edmunds, D. E., and W. D. Evans: Hardy operators, function spaces and embeddings. Springer, 2004.

[11] Fefferman, C., N. M. Rivière, and Y. Sagher: Interpolation between $H^{p}$ spaces: the real method. - Trans. Amer. Math. Soc. 191, 1974, 75-81.

[12] Frazier, M., and B. Jawerth: A discrete transform and decomposition of distribution spaces. - J. Funct. Anal. 93, 1990, 34-170.

[13] Gogatishvilli, A., B. Opic, and W. Trebels: Limiting reiteration for real interpolation with slowly varying functions. - Math. Nachr. 278, 2005, 86-107.

[14] Gustavsson, J., and J. Peetre: Interpolation of Orlicz spaces. - Studia Math. 60, 1977, $33-59$.

[15] Gustavsson, J.: A function parameter in connection with interpolation of Banach spaces. Math. Scand. 42, 1978, 289-305.

[16] Hedberg, L., and Y. Netrusov: An axiomatic approach to function spaces, spectral synthesis, and Luzin approximation. - Mem. Amer. Math. Soc. 188:882, 2007.

[17] Ho, K.-P.: Annihilator, completeness and convergence of wavelet system. - Nagoya Math. J. 188, 2007, 59-105.

[18] Ho, K.-P.: Littlewood-Paley spaces. - Math. Scand. 108, 2011, 77-102.

[19] Ho, K.-P.: Atomic decompositions, dual spaces and interpolations of martingale HardyLorentz-Karamata spaces. - Q. J. Math. 65, 2014, 985-1009.

[20] Ho, K.-P.: Sobolev-Jawerth embedding of Triebel-Lizorkin-Morrey-Lorentz spaces and fractional integral operator on Hardy type spaces. - Math. Nachr. 287, 2014, 1674-1686.

[21] Ho, K.-P.: Dual spaces of Littlewood-Paley spaces and Radon-Nikodým property. - Preprint.

[22] Holmstedt, T.: Interpolation of quasi-normed spaces. - Math. Scand. 26, 1970, 177-199.

[23] Jawerth, B.: Some observations on Besov and Lizorkin-Triebel spaces. - Math. Scand. 40, 1977, 94-104.

[24] Kalton, N., N. Peck, and J. Roberts: An F-space sampler. - London Math. Soc. Lecture Note Ser. 89, Cambridge Univ. Press, 1984.

[25] Krantz, S.: Fractional intgeration on Hardy spaces. - Studia Math. 63, 1982, 87-94.

[26] Liang, Y., Y. Sawano, T. Ullrich, D. Yang, and W. Yuan: A new framework for generalized Besov-type and Triebel-Lizorkin-type spaces. - Dissertationes Math. (Rozprawy Mat.) 489, 2013, 1-114.

[27] Lindenstrauss, J., and L. Tzafriri: Classical Banach spaces I and II. - Springer, 1996.

[28] Montgomery-Smith, S.: The Hardy operator and Boyd indices, interaction between functional analysis, harmonic analysis, and probability. - Marcel Dekker, Inc., 1996.

[29] Okada, S., W. Ricker, and E. SÁnchez Pérez: Optimal domain and integral extension of operators. - Birkhäuser, Basel, 2008.

[30] Sogge, C.: Fourier integrals in classical analysis. - Cambridge Univ. Press, 1993.

[31] Stein, E.: Harmonic analysis. - Princeton Univ. Press, 1993.

[32] Triebel, H.: Interpolation theory, function spaces, differential operators. - North-Holland, Amsterdam, New York, Oxford, 1978.

[33] Triebel, H.: Theory of function spaces. - Monographs in Math. 78, Birkhäuser, 1983.

[34] Viviani, B.: An atomic decomposition of the predual of $B M O(\rho)$. - Rev. Mat. Iberoam. 3, 1987, 401-425. 